\title{
Screening-Level Estimates of Mass Discharge Uncertainty from Point Measurement Methods
}

\author{
Michael C. Brooks ${ }^{1 *}$, Ki Young Cha ${ }^{2}$, A. Lynn Wood ${ }^{1}$ and Michael D. Annable ${ }^{3}$ \\ ${ }^{1}$ National Risk Management Research Laboratory \\ US Environmental Protection Agency \\ Ada, OK 74820 \\ ${ }^{2}$ National Research Council Post-Doctoral Associate \\ US Environmental Protection Agency \\ Ada, OK 74820 \\ ${ }^{3}$ Interdisciplinary Program in Hydrologic Sciences, \\ Department of Environmental Engineering Sciences \\ University of Florida \\ Gainesville, FL 32611 \\ *Corresponding author \\ Postal address: 919 Kerr Research Drive, Ada, OK 74820 \\ Phone number: 580/436-8982 \\ Email address: brooks.michael@epa.gov
}

Submitted for Publication in Journal of Contaminant Hydrology

November 2014

Revised March 2015 


\begin{abstract}
The uncertainty of mass discharge measurements associated with point-scale measurement techniques was investigated by deriving analytical solutions for the mass discharge coefficient of variation for two simplified, conceptual models. In the first case, a depth-averaged domain was assumed, consisting of one-dimensional groundwater flow perpendicular to a one-dimensional control plane of uniformly spaced sampling points. The contaminant flux along the control plane was assumed to be normally distributed. The second case consisted of one-dimensional groundwater flow perpendicular to a twodimensional control plane of uniformly spaced sampling points. The contaminant flux in this case was assumed to be distributed according to a bivariate normal distribution. The center point for the flux distributions in both cases was allowed to vary in the domain of the control plane as a uniform random variable. Simplified equations for the uncertainty were investigated to facilitate screening-level evaluations of uncertainty as a function of sampling network design. Results were used to express uncertainty as a function of the length of the control plane and number of wells, or alternatively as a function of the sample spacing. Uncertainty was also expressed as a function of a new dimensionless parameter, $\Omega$, defined as the ratio of the maximum local flux to the product of mass discharge and sample density. Expressing uncertainty as a function of $\Omega$ provided a convenient means to demonstrate the relationship between uncertainty, the magnitude of a local hot spot, magnitude of mass discharge, distribution of the contaminant across the control plane, and the sampling density.
\end{abstract}

Keywords: mass flux, mass discharge, uncertainty 


\section{INTRODUCTION}

Contaminant mass flux $J\left[\mathrm{ML}^{-2} \mathrm{~T}^{-1}\right]$ and mass discharge $\dot{m}\left[\mathrm{MT}^{-1}\right]$ combine two important features of contaminant risk: concentration $C\left[\mathrm{ML}^{-3}\right]$ and mobility (e.g., Suthersan et al., 2010). These measurements have been used for a number of site management purposes, including assessments of degradation rates (e.g., Semprini et al., 1995; Borden et al., 1997; King et al., 1999; Kao and Wang 2000; Kao and Prosser, 2001; Kao and Wang, 2001; Suarez and Rifia, 2002), characterization of source zones and associated plumes (e.g., King et al., 1999; Einarson and Mackay, 2001; Guilbeault et al., 2005; Basu et al., 2006; Fraser et al., 2008; Basu et al., 2009; Newell et al., 2011), characterization of back diffusion from aquitards (Chapman and Parker, 2005), and assessments of benefits from partial mass removal from DNAPL source zones (Brooks et al., 2008; DiFilippo and Brusseau, 2008; Cai et al., 2012).

Specific methods used to measure $J$ and $\dot{m}$ have been summarized in several publications (e.g., Kübert and Finkel, 2006; ITRC, 2010; Kavanaugh et al., 2011; Chen et al., 2014), and can be divided into two broad categories: point-measurement methods and pumping-measurement methods. Point-measurement methods are based on sampling techniques with relatively small sampling volumes, and most often consist either of applications based on multi-level samplers (e.g., Kao and Wang, 2001; Guilbeault et al., 2005; Freitas et al., 2011) or passive flux meters (PFMs) (e.g., Hatfield et al., 2004; Annable et al., 2005). With respect to the former, variations between approaches stem from methods used to estimate the Darcy flux $q\left[\mathrm{LT}^{-1}\right]$, and equally important, the spatial scale over which those measurements are made. A common criticism of pointmeasurement methods is the uncertainty that results when they are used to estimate $\dot{m}$ 
due to the un-sampled regions between measurement locations. In contrast, pumpingmeasurement methods integrate information over a much larger sampling volume, and thereby minimize the potential for uncertainty due to un-sampled regions between pointmeasurement locations. Pumping-measurement methods can be sub-divided into two groups: steady-state (Holder et al., 1998; Einarson and Mackay, 2001; Bayer-Raich et al., 2004; Brusseau et al., 2007) and transient methods. In the latter category, the original and most common pumping-measurement method is the integral pump test (Schwartz et al., 1998; Bockelmann et al., 2001; Bayer-Raich et al., 2004, Bayer-Raich et al., 2006), but other pumping-based approaches have been investigated and used (Brooks et al., 2008; Goltz et al., 2009; Kavanaugh et al., 2011).

The gain in certainty by minimizing un-sampled space in the application of a pumping-measurement method comes at the expense in loss of information about the spatial $J$ distribution. Thus, a trade-off is made between spatial information and the level of uncertainty associated with the measurement of $\dot{m}$. The uncertainty associated with un-sampled space when using a point measurement can of course be minimized by collecting more point measurements to reduce the distance between sampling locations. Increasing the number of samples, however, increases cost.

A number of studies have been completed on point-measurement method uncertainty (Kübert and Finkel, 2006; Li et al., 2007; Li and Abriola, 2009; Troldborg et al., 2010; Schwede and Cirpka, 2010; Béland-Pelletier et al., 2011; Cai et al., 2011; Cai et al., 2012; MacKay et al., 2012; Troldborg et al., 2012; Klammler et al., 2012; Chen et al., 2014). Two of these studies were based on field trials (Béland-Pelletier et al., 2011; MacKay et al., 2012), two of the studies used flow and transport simulations within 
Monte Carlo frameworks (Kübert and Finkel, 2006; Chen et al., 2014); two more studies likewise used flow and transport simulations within Monte Carlo frameworks, but simulations were conditioned to field data (Schwede and Cirpka, 2010; Troldborg et al., 2010); and the remaining studies employed various conditional, geostatistical techniques, wherein one or more parameters across the control plane were treated as spatial random variables.

Kubert and Finkel (2006) conducted an extensive Monte Carlo analysis on a hypothetical site to evaluate uncertainty as a function of measurement method, sampling density $\left(S_{D}\right)$, and hydraulic conductivity $(K)$ heterogeneity. As an example of the results obtained, the mean relative error was less than $10 \%$ for all levels of heterogeneity using an approach that directly measured flux with $S_{D}=5 \mathrm{pts} / \mathrm{m}^{2}$. When $S_{D}$ was reduced to $0.1 \mathrm{pts} / \mathrm{m}^{2}$, the mean relative error increased, and ranged from $\sim 30 \%$ to $\sim 60 \%$ for 0.25 $\leq \sigma_{\ln K}^{2} \leq 4.5$. Their research also noted the uncertainty that may result when combining measurements based on different support volumes, as for example when local scale measurements of $K$ are combined with site-wide average measurements of the hydraulic gradient $I$. Under this approach, the mean relative error ranged from approximately $40 \%$ to $500 \%$ for $0.25 \leq \sigma_{\ln K}^{2} \leq 4.5$, even using the highest sampling density of $5 \mathrm{pts} / \mathrm{m}^{2}$.

Kübert and Finkel (2006) used a temporally and spatially constant source of uniform $C$ to generate the contaminant plume in their simulations. The area of the source was $20 \%$ of the model domain cross section, and therefore was more than $20 \%$ of the control plane area. As a comparison, Guilbeault et al. (2005) noted that $80 \%$ of the plume mass-discharge occurred within $10 \%$ or less of the control plane area at three field sites they investigated. The impact of smaller contaminant mass distributions on 
uncertainty was investigated by Li et al. (2007), as part of a method they demonstrate to quantify uncertainty using empirical mass discharge cumulative distribution functions (CDFs) based on joint geostatistical simulations of random $C$ and $K$ fields conditioned to field measurements. They noted that in the case of $\dot{m}=319 \mathrm{~g} / \mathrm{d}$, a $S_{D}$ of $0.1 \mathrm{pt} / \mathrm{m}^{2}$ yielded a mean relative error of $\sim 20 \%$, but the same $S_{D}$ yielded a mean relative error of $\sim 180 \%$ in the case of $\dot{m}=15 \mathrm{~g} / \mathrm{d}$. To achieve a $\sim 20 \%$ mean relative error for $\dot{m}=15$ $\mathrm{g} / \mathrm{d}$, their results indicated a $S_{D}$ of $\sim 3 \mathrm{pt} / \mathrm{m}^{2}$ would be needed. This work was extended by $\mathrm{Li}$ and Abriola (2009) who presented a staged sampling strategy, where optimal sample locations were identified by evaluating initial sampling results from mean $C$, local random variable entropy, and $C$ conditional variance criterion. Compared to sampling densities based on a single sampling event with a regularly spaced grid, a sampling density of half or less was needed based on their staged approach.

Other studies that likewise presented methods to estimate $\dot{m}$ uncertainty using geostatistical simulations of random spatial variables conditioned to field measurements include Cai et al. (2011), Cai et al. (2012), Klammler et al. (2012), and Troldborg et al. (2012). In each case, uncertainty was quantified by generating empirical CDFs of $\dot{m}$. Cai et al. (2011) assumed uniform flow, such that uncertainty stemmed only from the spatial $C$ distribution. This approach was extended in Cai et al. (2012) to include $K$ as a second, independent spatial random variable. In both cases, uncertainty was summarized using boxplot representations of the CDFs. Data from the boxplots was used to calculate a normalized $95 \%$ confidence interval (defined as the difference between the $97.5 \%$ and $2.5 \%$ quantiles, divided by the $50 \%$ quantile), which ranged from $35 \%$ to $76 \%$ for the case studies investigated. Troldborg et al. (2012) calculated mass discharge uncertainty 
through Bayesian conditional simulations, where $C$ and $q$ were treated as independent random spatial variables, but $q$ was generated from a joint conditional simulation of $K$ and $I$. The method was applied to a field site with $\sigma_{\operatorname{lnK}}^{2}=1.4$, and using sampling networks with $S_{D}=\left\{\begin{array}{lll}0.32, & 0.05\end{array}\right\} \mathrm{pts} / \mathrm{m}^{2}$, they estimated $\dot{m}=\left\{\begin{array}{ll}12, & 21\end{array}\right\} \mathrm{g} / \mathrm{d}$ total chlorinated ethenes, respectively. The coefficient of variation $C V$ associated with these two estimates were $\{43 \%, 74 \%\}$, respectively. Klammler et al., (2012) present a stochastic simulation method conditioned to PFM measurements to estimate the CDF of $\dot{m}$ across the control plane. The method was used to estimate uncertainties associated with two PFM deployments: in the first case, $C V=16 \%$ for a site with $\dot{m}=777 \mathrm{~g} / \mathrm{d}$ trichloroethylene using a sampling network with $S_{D}=0.4 \mathrm{pts} / \mathrm{m}^{2}$; and in the second case, $C V=7 \%$ for a site with $\dot{m}=19 \mathrm{~g} / \mathrm{d}$ uranium using a sampling network with $S_{D}=2.8$ $\mathrm{pts} / \mathrm{m}^{2}$. Also presented were two approximate techniques to estimate uncertainty from PFM measurements that did not require conditional simulation, which were applicable when a sufficient number of data had been collected.

Schwede and Cirpka (2010) and Troldorg et al. (2010) likewise present methods to estimate $\dot{m}$ and its uncertainty using stochastic simulations conditioned to field data, but they both based their analysis on groundwater flow and contaminant transport simulations. Schwede and Cirpka (2010) used a Monte Carlo analysis, accounting for measurement uncertainty, in which $K$ was treated as a spatial random variable, and each realization was conditioned to measurements of $C$, hydraulic head, and $K$. They were specifically interested in the problem of estimating rates of contaminant degradation using $\dot{m}$ measurements at upgradient and downgradient control planes. As such, the method estimated mass discharge and its uncertainty through both independent control 
plane inversion, as well as joint inversion using data from both control planes. The relative errors between the true and measured $\dot{m}$ ranged from $1 \%$ to $61 \%$ for the cases

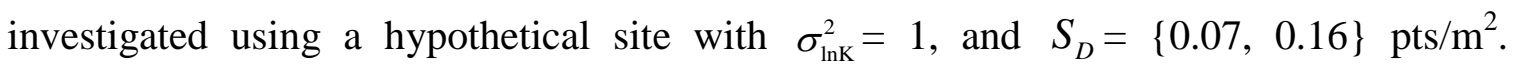
Troldborg et al. (2010) generated empirical mass discharge CDFs through Bayesian conditional simulations of flow and transport. Their approach accounted not only for subsurface heterogeneity and measurement uncertainty, but also conceptual model uncertainty through explicit consideration of alternative site conceptual models. A $C V$ of $54 \%$ was reported for the field case study presented, characterized by $\dot{m}=4.6 \mathrm{~g} / \mathrm{d}$ trichloroethylene, $S_{D}=0.02 \mathrm{pts} / \mathrm{m}^{2}$, and $\sigma_{\operatorname{lnK}}^{2}=0.3$.

Beland-Pelletier et al. (2011) provide a field-based analysis of uncertainty conducted at the Canadian Forces Base Borden, Ontario, Canada; where relative uncertainties in measurements, assumptions about $K$ and $I$, and uncertainty associated with $S_{D}$ were propagated to estimate the overall uncertainty in $\dot{m}$. Using a sampling network with $S_{D}=0.63 \mathrm{pts} / \mathrm{m}^{2}$, they estimated $\dot{m}=7.9 \mathrm{~g} / \mathrm{d}$ total hydrocarbons with a total uncertainty of $\sim 66 \%$. Mackay et al. (2012) also conducted a field-based evaluation of $\dot{m}$ uncertainty through a controlled release experiment of a bromide tracer at the Vandenberg Air Force Base, Santa Barbara, California. While the overall average injection rate was $122 \mathrm{~g} / \mathrm{d}$ bromide, true values of $\dot{m}$ at the control planes were assumed equal to the measurement completed with the highest sampling density $\left(S_{D}=1.4\right.$ pts $/ \mathrm{m}^{2}$ ), to account for temporal variations in $\dot{m}$ created by the flow field. Uncertainty, expressed as the relative percent difference between the true values and the estimates 
with sparser sampling networks, ranged from $-100 \%$ to $150 \%$ for sampling networks with $0.2 \leq S_{D} \leq 0.7 \mathrm{pts} / \mathrm{m}^{2}$

Collectively, the published literature has demonstrated that relatively large $\dot{m}$ uncertainty (>50\%) may result from subsurface heterogeneity, both in terms of heterogeneity in the flow field as well as heterogeneity in contaminant distribution. However, the uncertainty can be quantified once measurements are collected, and the uncertainty can be managed through the proper selection of measurement method and sampling density. With respect to an appropriate sampling density, we suggest there is a need for a more screening-level assessment of mass discharge uncertainty. A screening level assessment could be used to help guide preliminary field plans for mass discharge measurements. For example, such guidance could be used to help select sample spacing in the initial round of regularly-spaced sampling activities in the multi-staged sampling strategy of Li and Abriola (2009). Moreover, such an analysis is needed to help guide decisions about method selection when considering pumping-measurement versus pointmeasurement methods. The purpose of this paper is to present a screening level approach that can be used to help evaluate uncertainty when designing sampling networks for point-measurement methods. The analysis presented also provides a theoretical basis for highlighting the various factors that impact $\dot{m}$ measurement uncertainty.

\section{METHODS}

\subsection{Mass discharge measurement framework}


Consider a control plane of area $\Lambda\left[\mathrm{L}^{2}\right]$ oriented in the $y-z$ plane, the center of which corresponds to the origin, and which is normal to groundwater flow in the $+x$ direction. The advective contaminant mass flux at any point within the control plane is

$J=C q$

and, the $\dot{m}$ across the control plane is given by

$\dot{m}=\int_{\Lambda} J(y, z) d a$

As suggested by equation (2), $J$ is treated herein as a local measurement within the control plane, and $\dot{m}$ is considered a larger-scale measurement over the entire control plane. In practice, the function $J(y, z)$ is unknown and measurements are made at discrete locations across the control plane, in which case

$$
\dot{m}=\sum_{i=1}^{N} A_{i}^{*} J_{i}+\sum_{j=1}^{O-N} A_{j} \hat{J}_{j}
$$

where $A_{i}^{*}\left[\mathrm{~L}^{2}\right]$ is the support area (i.e., support volume per unit width in the flow direction) associated with the measured flux $J_{i}$ at the $i^{\text {th }}$ measurement point, $N$ is the number of discrete measurements, $O$ is the total number of subsections into which the control plane is divided, and $A_{j}\left[\mathrm{~L}^{2}\right]$ is the area associated with the interpolated flux value $\hat{J}_{j}$ at the $j^{\text {th }}$ un-sampled subsection. Values of $\hat{J}$ are estimated from a given interpolation scheme using measured $J$, and can include relatively simple approaches such as a Theissen Polygon approach (e.g., MacKay et al., 2012) to more complex Bayesian geostatisical approaches (e.g., Troldborg et al., 2012). Equation (3), while different from typical expressions of $\dot{m}$ based on discrete measurements, is useful for illustrating two sources of uncertainty associated with $\dot{m}$ : uncertainty associated with the 
actual measurements of $J$ at $N$ locations (first term on the right-hand side, directmeasurement uncertainty), and interpolation uncertainty associated with estimating $\hat{J}$ across the rest of the un-sampled control plane (second term on the right-hand side). Direct measurement uncertainty has been discussed elsewhere (e.g., Wood et al., 2009; Béland-Pelletier et al., 2011; Troldborg et al., 2012), and our focus in this screening analysis is the interpolation uncertainty because it is considered to represent the greater source of uncertainty (e.g., Béland-Pelletier et al., 2011). However, this conclusion should be evaluated on a case-by-case basis to ensure direct measurement uncertainty is a secondary concern to interpolation uncertainty.

It is also important to recognize that there are features associated with the control plane itself that may impact $\dot{m}$ measurements. Control plane features include the horizontal and vertical extent, its orientation relative to the groundwater flow direction (which may be temporally variable, e.g., Rein et al., 2009), its distance from the source zone, and the internal horizontal and vertical sample spacing. The first two features are generally selected based on prior characterization information, and the third is dependent on the purpose for collecting $\dot{m}$ measurements. These three aspects of the control plane are not discussed further here. The fourth feature (sample spacing) controls interpolation uncertainty, and is the sole consideration in this screening analysis.

\subsection{Screening-Level Uncertainty Equations}

To develop screening-level equations for interpolation uncertainty, it is assumed that the flux distribution associated with a contaminant plume intersecting the control 
plane is described by an uncorrelated bivariate normal distribution centered on $y=\mu_{y}$

$[\mathrm{L}]$ and $z=\mu_{z}[\mathrm{~L}]$ :

$J(y, z)=\frac{S_{2}}{2 \pi \sigma_{y} \sigma_{z}} \exp \left[-\frac{1}{2}\left(\frac{\left(y-\mu_{y}\right)^{2}}{\sigma_{y}^{2}}+\frac{\left(z-\mu_{z}\right)^{2}}{\sigma_{z}^{2}}\right)\right]$,

where $S_{2}\left[\mathrm{MT}^{-1}\right]$ is a scaling factor that reflects the maximum contaminant flux $J_{\max }$ at $\left(\mu_{y}, \mu_{z}\right)$, and $\sigma_{y}$ and $\sigma_{z}[\mathrm{~L}]$ are spatial standard deviations of the distribution along the $y$ and $z$ axes, respectively. The $J$ is assumed constant along the $x$ axis in the vicinity of the control plane; therefore $J$ varies only in the $y-z$ plane. At $\left(\mu_{y}, \mu_{z}\right)$, $J_{\max }=S_{2} /\left(2 \pi \sigma_{y} \sigma_{z}\right)$, or $S_{2}=2 \pi \sigma_{y} \sigma_{z} J_{\max }$. Substituting equation (4) into equation (2) yields

$\dot{m}_{2}=\int_{z_{1}}^{z_{2}} \int_{y_{1}}^{y_{2}} \frac{S_{2}}{2 \pi \sigma_{y} \sigma_{z}} \exp \left[-\frac{1}{2}\left(\frac{\left(y-\mu_{y}\right)^{2}}{\sigma_{y}^{2}}+\frac{\left(z-\mu_{z}\right)^{2}}{\sigma_{z}^{2}}\right)\right] d y d z$

where $\dot{m}_{2}$ shall be used from this point forward to represent mass discharge across a twodimensional control plane, as opposed to $\dot{m}$ which shall be used to represent mass discharge across a depth-averaged, one-dimensional control plane (as discussed further in the following sections). If the limits of integration are large enough relative to $\sigma_{y}$ and $\sigma_{z}$, the integration expressed in equation (5) results in

$\dot{m}_{2 T}=2 \pi \sigma_{y} \sigma_{z} J_{\max }$

where $\dot{m}_{2 T}$ is the mass discharge of the entire two-dimensional plume. In this formulation, $S_{2}=\dot{m}_{2 T}$. 
Alternatively, a depth-averaged, one-dimensional version of equation (4) can be

used:

$\bar{J}(y)=\frac{S_{1}}{\sigma_{y} \sqrt{2 \pi}} \exp \left[-\frac{1}{2}\left(\frac{y-\mu_{y}}{\sigma_{y}}\right)^{2}\right]$,

where $S_{1}=\sqrt{2 \pi} \sigma_{y} \bar{J}_{\max }$, and $\bar{J}_{\max }\left[\mathrm{MT}^{-1} \mathrm{~L}^{-2}\right]$ is the depth-averaged maximum contaminant flux at $y=\mu_{y}$. Under this condition, the equivalent expression to equation (6) is

$\dot{m}_{T}=\sqrt{2 \pi} B \sigma_{y} \bar{J}_{\max }$,

where $\dot{m}_{T}$ is the mass discharge of the entire one-dimensional, depth-averaged plume, and $B$ is aquifer depth [L]. This approach is similar to that used by Chen et al. (2014), who assumed $C$ varied only in the y-direction according to a normal distribution within a homogeneous $K$ field.

Expressing $J$ directly as either a bivariate normal distribution in equation (4) or a univariate normal distribution in equation (7) allows for mildly heterogeneous cases in unconsolidated geologic formations where local $J$ is influenced by $q$, but lateral spreading during travel from the source to the control plane is sufficient to create symmetric contaminant distributions about the hot spot. The conceptual model represented by these equations is intended to represent a challenging mass discharge measurement problem: a single hotspot in an otherwise uncontaminated region. The assumed distribution is not universally applicable to all possible subsurface $J$ distributions. In general, use of the assumed spatially continuous $J$ distribution across spatially discontinuous hydrogeologic media or highly heterogeneous media may not be applicable due to the 
potential for asymmetric or discontinuous $J$ distributions in those types of settings. However, in cases where discrete units can be considered separately, consideration could be given to applying the results herein separately to each individual hydrogeologic unit. Other conceptual models for the flux distribution could be used, but the normal distribution was generally considered most applicable. In addition, any conceptual model that assumes flux is more widely distributed (for example, assuming multiple hot spots) should reduce the uncertainty in $\dot{m}$ estimates. For example, Troldborg et al. (2012) reported a decrease in $\dot{m}$ uncertainty as the control plane distance from the source increased due to greater lateral transport from transverse dispersion. Chen et al. (2014) also noted a decrease in $\dot{m}$ uncertainty as the plume width increased relative to the well spacing.

\subsubsection{One-Dimensional Control Plane Analysis}

Consider a simplified, one-dimensional depth-averaged control plane consisting of $n \geq 2$ monitoring wells, as shown in Figure 1 which illustrates the specific case of $n=$ 7. The spacing between wells is $W[\mathrm{~L}]$, and the end wells in the control plane are located at $(0, b)$ and $(0,-b)$; therefore $(n-1) W=2 b$. The control plane is defined to extend a distance of $W / 2$ on each end, therefore the total length of the control plane $L_{C P}$ is $n W$. Contaminant flux across the control plane is described by equation (7). To represent uncertainty due to an unknown value of $\mu_{y}$ in a static flow field, or due to $\mu_{y}$ changing position as a result of a directionally variable flow field, $\mu_{y}$ is assumed to be a uniform random variable with limits $-b \leq \mu_{y} \leq b$. While $\sigma_{y}$ could also be treated as a stochastic 
variable, it will be treated as a deterministic variable to aid in the development of a screening-level equation. The assumption that $-b \leq \mu_{y} \leq b$ reflects a goal to have sufficient characterization information prior to the installation of the control plane to ensure the plume core is located within the limits of the control plane. Using the "measured" flux at the $n$ wells, $\dot{m}$ is estimated as

$\dot{m}\left(\mu_{y}\right)=W B \sum_{i=1}^{n} J\left(a_{i}\right)$

where $a_{i}$ is the $y$ coordinate for the $i^{\text {th }}$ well. The mean mass discharge $\widehat{\dot{m}}$ is given by

$\hat{\dot{m}}=E\left[\dot{m}\left(\mu_{y}\right)\right]=\int_{-b}^{b} \dot{m}(\mu) f(\mu) d \mu$,

where $f(\mu)$ is the probability density function for a uniform random variable $\mu_{y}$ :

$f\left(\mu_{y}\right)=\frac{1}{2 b}$

Integration of equation (10) after substitution of equations (9) and (11) results in

$$
\hat{\dot{m}}=\frac{B S_{1}}{2(n-1)} \sum_{i=1}^{n}\left[\operatorname{erf}\left(\frac{a_{i}+b}{\sigma_{y} \sqrt{2}}\right)-\operatorname{erf}\left(\frac{a_{i}-b}{\sigma_{y} \sqrt{2}}\right)\right] \text {, }
$$

which can alternatively be expressed as

$$
\widehat{\dot{m}}=\frac{B S_{1}}{(n-1)} \sum_{i=1}^{n-1} \operatorname{erf}\left(i \frac{\xi}{\sqrt{2}} \frac{b}{\sigma_{y}}\right) \text {, }
$$

where $\xi=2 /(n-1)$, and is the coefficient which equates $W$ to $b$ (i.e., $W=\xi b)$. The variance of mass discharge $\sigma_{\dot{m}}^{2}$ is given by

$$
\sigma_{\dot{m}}^{2}=\int_{-b}^{b}[\dot{m}(\mu)]^{2} f(\mu) d \mu-\{\hat{\dot{m}}\}^{2}
$$

which, upon substitution of equations (9) and (11) into (14) results in 


$$
\sigma_{\dot{m}}^{2}=\left(\frac{W B S_{1}}{\sigma_{y} \sqrt{2 \pi}}\right)^{2}\left(\frac{1}{2 b}\right) \int_{-b}^{b}\left\{\sum_{i=1}^{n} \exp \left[-\frac{1}{2}\left(\frac{a_{i}-\mu}{\sigma_{y}}\right)^{2}\right]\right\}^{2} d \mu-(\hat{\dot{m}})^{2} .
$$

It is helpful to note that the argument of the integral in equation (15) can be expressed as

$$
\begin{aligned}
& \left\{\sum_{i=1}^{n} \exp \left[-\frac{1}{2}\left(\frac{a_{i}-\mu}{\sigma_{y}}\right)^{2}\right]\right\}^{2}= \\
& \sum_{i=1}^{n}\left\{\exp \left[-\frac{1}{2}\left(\frac{a_{i}-\mu}{\sigma_{y}}\right)^{2}\right]\right\}^{2}+2 \sum_{i=1}^{n} \sum_{j>i}^{n} \exp \left[-\frac{1}{2}\left(\frac{a_{i}-\mu}{\sigma_{y}}\right)^{2}\right] \exp \left[-\frac{1}{2}\left(\frac{a_{j}-\mu}{\sigma_{y}}\right)^{2}\right]
\end{aligned}
$$

Integrating the first term on the right-hand side yields

$$
\int_{-b}^{b} \sum_{i=1}^{n}\left\{\exp \left[-\frac{1}{2}\left(\frac{a_{i}-\mu}{\sigma_{y}}\right)^{2}\right]\right\}^{2} d \mu=\frac{1}{2} \sqrt{\pi} \sigma_{y} \sum_{i=1}^{n}\left\{\operatorname{erf}\left(\frac{a_{i}+b}{\sigma_{y}}\right)-\operatorname{erf}\left(\frac{a_{i}-b}{\sigma_{y}}\right)\right\},
$$

and integrating the second term on the right hand side yields

$$
\begin{aligned}
& \int_{-b}^{b} 2 \sum_{i=1}^{n} \sum_{j>i}^{n} \exp \left[-\frac{1}{2}\left(\frac{a_{i}-\mu}{\sigma_{y}}\right)^{2}\right] \exp \left[-\frac{1}{2}\left(\frac{a_{j}-\mu}{\sigma_{y}}\right)^{2}\right] d \mu= \\
& \sqrt{\pi} \sigma_{y} \sum_{i=1}^{n} \sum_{j>i}^{n} \exp \left[-\left(\frac{a_{i}-a_{j}}{2 \sigma_{y}}\right)^{2}\right]\left[\operatorname{erf}\left(\frac{a_{i}+a_{j}+2 b}{2 \sigma_{y}}\right)-\operatorname{erf}\left(\frac{a_{i}+a_{j}-2 b}{2 \sigma_{y}}\right)\right]
\end{aligned}
$$

Therefore, the variance is

$$
\sigma_{\dot{m}}^{2}=\frac{1}{2 \sqrt{\pi}}\left(\frac{B S_{1}}{n-1}\right)^{2} \frac{b}{\sigma_{y}}\left\{\begin{array}{l}
\sum_{i=1}^{n}\left[\operatorname{erf}\left(\frac{a_{i}+b}{\sigma_{y}}\right)-\operatorname{erf}\left(\frac{a_{i}-b}{\sigma_{y}}\right)\right]+ \\
2 \sum_{i=1}^{n} \sum_{j>i}^{n} \exp \left[-\left(\frac{a_{i}-a_{j}}{2 \sigma_{y}}\right)^{2}\right]\left[\operatorname{erf}\left(\frac{a_{i}+a_{j}+2 b}{2 \sigma_{y}}\right)-\operatorname{erf}\left(\frac{a_{i}+a_{j}-2 b}{2 \sigma_{y}}\right)\right]
\end{array}\right\}-(\hat{\dot{m}})^{2}
$$

Equation (19) can also be expressed as 


$$
\sigma_{\dot{m}}^{2}=\frac{1}{\sqrt{\pi}}\left(\frac{B S_{1}}{n-1}\right)^{2} \frac{b}{\sigma_{y}}\left\{\begin{array}{l}
\sum_{i=1}^{n-1} \operatorname{erf}\left(i \xi \frac{b}{\sigma_{y}}\right)+ \\
2 \sum_{i=1}^{n-1}\left\{\exp \left[-\left(i \frac{\xi}{2} \frac{b}{\sigma_{y}}\right)^{2}\right] \sum_{j=i}^{n-1} \operatorname{erf}\left((2 j-i) \frac{\xi}{2} \frac{b}{\sigma_{y}}\right)\right\}
\end{array}\right\}-(\hat{\dot{m}})^{2} .
$$

Finally, the $\dot{m}$ coefficent of variation $C V_{\dot{m}}$, is defined as the ratio $\sigma_{\dot{m}} / \widehat{\dot{m}}$ :

$$
C V_{\dot{m}}=\sqrt{\frac{b}{\sqrt{\pi} \sigma_{y}} \frac{1}{\left.\sum_{i=1}^{n-1} \operatorname{erf}\left(\frac{i \xi b}{\sqrt{2} \sigma_{y}}\right)\right]^{2}}\left\{\sum_{i=1}^{n-1} \operatorname{erf}\left(\frac{i \xi b}{\sigma_{y}}\right)+2 \sum_{i=1}^{n-1}\left\{\exp \left[-\left(\frac{i \xi b}{2 \sigma_{y}}\right)^{2}\right] \sum_{j=i}^{n-1} \operatorname{erf}\left((2 j-i) \frac{\xi b}{2 \sigma_{y}}\right)\right\}\right\}-1}
$$

As shown in equation (21), $C V_{\dot{m}}$ is only a function of $n$ and the ratio $b / \sigma_{y}$, which is a measure of the length of the control plane relative to the plume width.

\subsubsection{Two-Dimensional Control Plane Analysis}

The derivations above for the depth-averaged, one-dimensional control plane can be expanded to consider monitoring points distributed across a two-dimensional control plane. In general, for a regularly spaced sampling network with $n$ horizontal locations and $p$ vertical locations, the measured mass discharge across the two-dimensional control plane is

$$
\dot{m}_{2}=W Z \sum_{i=1}^{n} \sum_{j=1}^{p} J\left(a_{i}^{h}, a_{j}^{v}\right)
$$

where $Z[\mathrm{~L}]$ is the vertical distance between sampling locations, $p$ is the number of vertical sampling locations, and $J\left(a_{i}^{h}, a_{j}^{v}\right)$ is the flux given by equation (4) at location 
$\left(a_{i}^{h}, a_{j}^{v}\right)$. To facilitate the derivation of an analytical equation, assume the sampling network consists of 4 locations as illustrated in Figure 2, in which case

$$
\dot{m}_{2}=4 b d[J(b, d)+J(b,-d)+J(-b,-d)+J(-b, d)]
$$

Similar to the previous case, the control plane is defined to extend beyond the location of the four points by a distance of $W / 2$ each way in the horizontal and $Z / 2$ each way in the vertical. The center of the plume $\left(\mu_{y}, \mu_{z}\right)$ is assumed to be a bivariate, uniform random variable in the region $-b \leq \mu_{y} \leq b$ and $-d \leq \mu_{z} \leq d$, which as in the one-dimensional control plane case, represents uncertainty in the plume position or changing plume position. Similar to the horizontal case, the relationship between the vertical spacing between sampling locations $Z[\mathrm{~L}]$ and $d$ is $(p-1) Z=2 d$. The terms $\sigma_{y}$ and $\sigma_{z}$ are treated as deterministic variables. The mean mass discharge $\hat{\dot{m}}_{2}$ is given by

$$
\hat{\dot{m}}_{2}=E\left[\dot{m}_{2}\left(\mu_{y}, \mu_{z}\right)\right]=\int_{-d}^{d} \int_{-b}^{b} \dot{m}_{2}\left(\mu_{1}, \mu_{2}\right) g\left(\mu_{1}, \mu_{2}\right) d \mu_{1} d \mu_{2},
$$

where $g\left(\mu_{y}, \mu_{z}\right)$ is the probability density function for the bivariate uniform random variable:

$$
g\left(\mu_{y}, \mu_{z}\right)=\frac{1}{4 b d}
$$

Integration of equation (24) after substitution of equations (23) and (25) results in

$$
\widehat{\dot{m}}_{2}=S_{2} \operatorname{erf}[\sqrt{2} \beta] \operatorname{erf}[\sqrt{2} \delta]
$$

where $\beta=b / \sigma_{y}$ and $\delta=d / \sigma_{z}$. The variance of $\dot{m}_{2}$ is given by

$$
\sigma_{\dot{m}[2]}^{2}=\int_{-d}^{d} \int_{-b}^{b}\left[\dot{m}_{2}\right]^{2} g\left(\mu_{1}, \mu_{2}\right) d \mu_{1} d \mu_{2}-\left\{\hat{\dot{m}}_{2}\right\}^{2}
$$


which, upon substitution of equations (23) and (25) for $\dot{m}_{2}$ and $g\left(\mu_{y}, \mu_{z}\right)$ respectively, results in

$$
\sigma_{\dot{m}[2]}^{2}=\frac{S_{2}^{2} \beta \delta}{\pi}\left\{\begin{array}{l}
\operatorname{erf}(2 \beta) \operatorname{erf}(2 \delta)+ \\
2 \exp \left(-\delta^{2}\right) \operatorname{erf}(2 \beta) \operatorname{erf}(\delta)+ \\
2 \exp \left(-\beta^{2}\right) \operatorname{erf}(\beta) \operatorname{erf}(2 \delta)+ \\
4 \exp \left(-\beta^{2}\right) \exp \left(-\delta^{2}\right) \operatorname{erf}(\beta) \operatorname{erf}(\delta)
\end{array}\right\}-\left\{\widehat{\dot{m}}_{2}\right\}^{2}
$$

The coefficient of variation in the case of this two-dimensional control plane $C V_{\dot{m}[2]}$ is equal to the ratio of equations (28) and (26):

$$
C V_{\dot{m}[2]}=\left\{\frac{\beta \delta}{\pi[\operatorname{erf}(\sqrt{2} \beta) \operatorname{erf}(\sqrt{2} \delta)]^{2}}\left\{\begin{array}{l}
\operatorname{erf}(2 \beta) \operatorname{erf}(2 \delta)+ \\
2 \exp \left(-\delta^{2}\right) \operatorname{erf}(2 \beta) \operatorname{erf}(\delta)+ \\
2 \exp \left(-\beta^{2}\right) \operatorname{erf}(\beta) \operatorname{erf}(2 \delta)+ \\
4 \exp \left(-\beta^{2}\right) \exp \left(-\delta^{2}\right) \operatorname{erf}(\beta) \operatorname{erf}(\delta)
\end{array}\right\}-1\right\}^{\frac{1}{2}},
$$

and is a function of $b / \sigma_{y}$ and $d / \sigma_{z}$ only since this derivation is specific for $n=p=2$.

An analytical solution for the two-dimensional case with more than 4 points was not investigated. However, for comparison results for cases with more than 4 points were generated numerically using a Monte Carlo analysis implemented in a Matlab script. For each value of $n=p=\{2,4,6,8,10\}$, Monte Carlo analyses were completed for a range of $\sigma_{y}$ and $\sigma_{z}$ values such that $0 \leq b / \sigma_{y}=d / \sigma_{z} \leq 10$. Within each realization, equation (22) was used to estimate $\dot{m}_{2}$, where $\mu_{y}$ and $\mu_{z}$ were selected as uniform random variables within the domain of $b=d=10 \mathrm{~m}$. Equations (24) and (27) were then approximated by taking the average and standard deviation of $\dot{m}_{2}$ across all realizations of a given Monte 
Carlo analysis. The number of realizations completed was 100,000, and no significant difference in results was noted in test trials using $1,000,000$ realizations.

\section{RESULTS and DISCUSSION}

\subsection{One-Dimensional Control Plane Analysis}

Plotted in Figure 3a is $C V_{\dot{m}}$ as a function of $b / \sigma_{y}$ for the point-measurement method based on equation (21) for $2 \leq n \leq 10$. The insert graphic shows the same information on a $\log$-linear basis. In the region where $C V_{\dot{m}}$ is larger than roughly $10 \%$, the figures illustrate that $C V_{\dot{m}}$ increases as $b / \sigma_{y}$ increases, reflecting the growing uncertainty of trying to quantify $\dot{m}$ associated with a relatively narrow plume using a relatively wide control plane; and $C V_{\dot{m}}$ decreases as $b / \sigma_{y}$ decreases, because in the limit as $\sigma_{y} \rightarrow \infty, J$ becomes uniformly distributed across the control plane and no variation in $\dot{m}$ is expected due to variations in $\mu_{y}$. Likewise, as $n$ increases, $C V_{\dot{m}}$ decreases as expected. Assuming that the width of the contaminant plume can be defined as $\pm 3 \sigma_{y}$, and that $L_{C P}$ is sized to cover the plume such that $2 b=6 \sigma_{y}$; then $b / \sigma_{y}=3$ and $C V_{\dot{m}}<$ $\sim 15 \%$ for $n \geq 3$. However, if the plume width is reduced by half, then $b / \sigma_{y}=6$, and $n \geq 5$ is required to achieve a comparable level of certainty. Likewise, if the plume width is one third of $2 b$, then $b / \sigma_{y}=9$, and $n \geq 7$ to achieve a comparable level of certainty.

In the region of $C V_{\dot{m}}<\sim 15 \%$, the functionality between $C V_{\dot{m}}, b / \sigma_{y}$, and $n$ is more complex. Local minimums and maximums are evident, as highlighted by the insert 
graphic in Figure 3a. Moreover, the minima and maxima values are a function of $n$, with the difference between the two increasing as $n$ increases. This contradicts an expectation that $C V_{\dot{m}}$ should decrease uniformly as $n$ increases. Some of the complexity represented in Figure 3a can be reduced if $C V_{\dot{m}}$ is expressed as a function of $W / \sigma_{y}$ as illustrated in Figure $3 b$. In this case, the curves plot on top of each other for $W / \sigma_{y}>2.2$, a single local minimum is evident at $W / \sigma_{y}=2.2$, and differences in $C V_{\dot{m}}$ as a function of $n$ are evident for $W / \sigma_{y}<2.2$. The local $C V_{\dot{m}}$ maxima noted in Figure 3a are likewise evident in Figure $3 \mathrm{~b}$ for $W / \sigma_{y}<2.2$, and are likewise proportional to $n$. This reflects the condition that a smaller number of wells (and hence more widely distributed) provides a less variable estimate of $\dot{m}$ as $\mu_{y}$ varies across the control plane for the condition of a wide plume (i.e., small $W / \sigma_{y}$ ). For a larger number of wells, $\dot{m}$ varies more as $\mu_{y}$ varies across the control plane. This is illustrated in Figure $4 \mathrm{a}$ where measured $\dot{m}$ is plotted as a function of $\mu_{y}$ for $n=\{2,10\}$ and $W / \sigma_{y}=0.4$, which qualitatively represents a wide plume. For comparison, Figure $4 \mathrm{~b}$ shows measured $\dot{m}$ for the same well configurations when the plume is narrow relative to well spacing $\left(W / \sigma_{y}=4\right)$. For the narrow plume, the variations in measured $\dot{m}$ are similar for both well spacings, consequently no variation in $C V_{\dot{m}}$ is noted when plotted as a function of $W / \sigma_{y}$ (i.e., Figure $3 \mathrm{~b}$ for $W / \sigma_{y}>2.2$ ).

For the conditions represented in Figure $4, \dot{m}_{T}=10 \mathrm{~g} / \mathrm{d}$. For the wide-plume case (Figure 4a) $\hat{\dot{m}}=\{3.1,8.3\} \mathrm{g} / \mathrm{d}$ for $n=\{2,10\}$, respectively. When a significant portion of the plume consistently extends beyond the ends of the control plane (as in this wide-plume case), $\dot{m}_{T}$ and $\hat{\dot{m}}$ differ and the result is measurement bias. Consequently, 
it is important to note that the case of $n=2$ in Figure 4a does not provide a better estimate of $\dot{m}_{T}$ compare to the case of $n=10$, even though the variability is less. The case of $n=$ 2 has more bias because a greater portion of the plume extends beyond the control plane. In contrast, for the narrow plume (Figure 4b), $\stackrel{\dot{m}}{\text { equals }} \dot{m}_{T}$ in both the $n=2$ and $n=10$ cases. These results of course reflect the issue of accuracy, whereas the emphasis placed in this paper is one of precision. Evaluating the accuracy of $\dot{m}$ can only be accomplished when $\dot{m}_{T}$ is known. When evaluating field measurements, $\dot{m}_{T}$ is unknown and the quality of the measurement is therefore evaluated using an assessment of its variability. Focusing solely on variability and ignoring potential bias is not viewed as a significant limitation of the proposed equations for screening-level uncertainty evaluations given the relatively small values of $C V_{\dot{m}}$ for which this behavior is noted (i.e., $C V_{\dot{m}}<\sim 15 \%$ ). If accurate quantification of relatively small uncertainties is needed, other more rigorous approaches should be used.

For $W / \sigma_{y} \geq 2.2$, Figure $3 b$ demonstrates that point-measurement methods provide a robust estimate of $\dot{m}$ only if $W$ is appropriate for the scale of subsurface heterogeneity, which in this case is represented by $\sigma_{y}$. An approximate, simplified expression can be obtained noting the limiting behavior of the exponential and error function terms in equation (21) as their arguments increase. Using $W / \sigma_{y}$ in place of $b / \sigma_{y}$, the simplified expression is

$$
C V_{\dot{m}} \cong \sqrt{\frac{W}{2 \sqrt{\pi} \sigma_{y}}-1}
$$


This approximate formula is plotted in Figure 3b, along with the percent error between it and equation (21) expressed as a function of $W / \sigma_{y}$. For $W / \sigma_{y} \geq 4$, corresponding to $C V_{\dot{m}} \cong 40 \%$, the absolute value of the percent error in the approximate formula is $13 \%$ or less, and for $W / \sigma_{y}>5$, the approximate expression gives essentially identical results to the true formula. The approximate equation does not, however, capture the minima and maxima details that occur for $W / \sigma_{y}<2.2$. Equation (30) should therefore be used to predict $C V_{\dot{m}} \geq 40 \%$, which is still considered useful as a screening-level tool.

It is instructive to recast $W / \sigma_{y}$ in an alternative form using a modified version of $S_{D}$, defined as

$$
S_{D}^{\prime}=\frac{1}{W B}
$$

where $S_{D}^{\prime}$ indicates the sampling density associated with a depth-averaged flux measurement over $B$. Using equation (8),

$$
\frac{W}{\sigma_{y}}=\sqrt{2 \pi} \Omega^{\prime}
$$

where $\Omega^{\prime}=\bar{J}_{\max } / \dot{m} S_{D}^{\prime}$; and equation (30) can be written as

$$
C V_{\dot{m}} \cong \sqrt{\frac{\Omega^{\prime}}{\sqrt{2}}-1}
$$

The terms $W / \sigma_{y}$ and $\Omega^{\prime}$ are linearly related, and $\Omega^{\prime}$ is shown as a second $x$-axis at the top of the graph in Figure $3 b$. Using $\Omega^{\prime}, C V_{\dot{m}}$ is readily seen as a function of three elemental aspects of $\dot{m}$ measurement. First, $C V_{\dot{m}}$ is inversely proportional to $S_{D}^{\prime}$, which expresses the intuitive relationship that a greater number of sampling points can improve 
the certainty of the measurement. Second, $C V_{\dot{m}}$ is proportional to $\bar{J}_{\max }$, which denotes that uncertainty increases with increasing potential for a local hot spot. Third, $C V_{\dot{m}}$ is inversely proportional to $\dot{m}$, which expresses the intuitive relationship that it is more difficult to quantify small values of $\dot{m}$ relative to large values of $\dot{m}$. Alternatively, the ratio $\dot{m} / \bar{J}_{\max }$ is an indication of the distribution of contaminant about the control plane, and uncertainty is inversely proportional to the extent of distribution.

\subsection{Two-Dimensional Control Plane Analysis}

Plotted in Figure 5a is $C V_{\dot{m}[2]}$ as a function of $b / \sigma_{y}=d / \sigma_{z}$ for $n=p=\{2,4,6$, $8,10\}$. Results for $n>2$ were obtained using Monte Carlo simulations, while results for $n=2$ were obtained using both Monte Carlo simulations and equation (29). The result is qualitatively similar to the one-dimensional control plane case discussed earlier: as $b / \sigma_{y}$ and $d / \sigma_{z}$ increase, so does $C V_{\dot{m}[2]}$, reflecting greater uncertainty as the size of the control plane increases relative to the plume dimensions. However, $C V_{\dot{m}[2]}$ is larger for values of $b / \sigma_{y}$ and $n$ compared to $C V_{\dot{m}}$, which is to be expected given the two spatial degrees of freedom over which the plume center point is allowed to vary. For comparison, Figure 5b shows $C V_{\dot{m}}$ calculated with equation (21) with $n=2$ and $C V_{\dot{m}[2]}$ calculated from equation (29) using $d / \sigma_{z}=\left\{2 b / \sigma_{y}, b / \sigma_{y}, b / 2 \sigma_{y}, b / 4 \sigma_{y}\right\}$. The uncertainty expressed using $C V_{\dot{m}[2]}$ approaches $C V_{\dot{m}}$ as $d / \sigma_{z}$ decreases, illustrating that the two-dimensional control plane case reduces to the one-dimensional, depth averaged case when a sufficient number of vertical samples are collected to accurately define the 
depth-averaged $J$. Similar to the one-dimensional control plane case, the figure illustrates that as the number of sampling locations in the control plane increases, $C V_{\dot{m i[2]}}$ decreases as expected. For example, when $b / \sigma_{y}=d / \sigma_{z}=5, C V_{\dot{m}[2]}$ is reduced from 2.6 to 0.34 (roughly 90\%) when the number of samples is increased from 4 to 16; likewise, it is reduced from 0.34 to 0.03 (roughly $90 \%$ again) as the number of samples is increased from 16 to 36.

Similar to the one-dimensional control plane case, $C V_{\text {mi[ }[2]}$ can be expressed as a function of $W / \sigma_{y}=Z / \sigma_{z}$ (Figure 6a), or as a function of $\Omega$ (Figure 6b), defined in this two-dimensional control plane case as $\Omega=\frac{J_{\max }}{\dot{m} S_{D}}$

These transformations are completed noting the functionality between $b$ and $W, d$ and $Z$, and $\sigma_{y} \sigma_{z}$ and $\dot{m}$, which give:

$\frac{b}{\sigma_{y}} \frac{d}{\sigma_{z}}=\frac{(n-1)(p-1)}{4} \frac{W}{\sigma_{y}} \frac{Z}{\sigma_{z}}=\frac{\pi}{2}(n-1)(p-1) \Omega$.

Results for $C V_{\dot{m}[2]}$, which plotted as separate curves for specific values of $n=p$ in Figure 5a, now coincide for $\Omega>\sim 0.5$ (or $W / \sigma_{y}=Z / \sigma_{z}>\sim 2$ ). Note that $C V_{\dot{m}[2]}$ monotonically increases as a function of $\Omega$ for $\Omega>1$. In this region, equation (34) can be used to make several observations about the dynamics of $C V_{\dot{m}[2]}, J_{\max }, \dot{m}$, and $S_{D}$. First, this formulation is consistent with previous observations regarding the inverse relationship between uncertainty and $S_{D}$. Moreover, it is also consistent with previous observations regarding the inter-relationship between $\dot{m}$ and $S_{D}$, i.e., as $\dot{m}$ decreases, $S_{D}$ 
needs to increase to maintain the same $C V_{\dot{m}[2]}$. This fact was noted by Li et al. (2007), who pointed out that $\dot{m}$ uncertainty may increase after remediation when trying to measure a smaller $\dot{m}$ with the same monitoring network. Likewise, the results here caution against defining an acceptable $S_{D}$ without regard to the magnitude of $\dot{m}$, especially given the potential range over which $\dot{m}$ may vary (e.g., Newell et al., 2011). However, this is true only if $J_{\max }$ is constant. If $\dot{m}$ decreases in proportion to $J_{\max }$, then no change in $S_{D}$ is needed to maintain $C V_{\dot{m}[2]}$. A similar result was noted by Basu et al. (2008), who evaluated with modeling simulations changes in source zone $J$ resulting from the dissolution of a DNAPL source. They found that the spatial coefficient of variation of $J$ was constant with DNAPL mass removal. Under these conditions and assuming a static flow field, changes in $\dot{m}$ and $J_{\max }$ are proportional, and therefore $C V_{\dot{m}}$ will remain the same using a constant $S_{D}$.

Similar to equation (33), an approximate solution for $C V_{\dot{m}[2]}$ can be obtained noting the limiting values for the exponential and error functions as the arguments increase in equation (29):

$$
C V_{\dot{m}[2]} \cong \sqrt{\frac{\Omega}{2}-1} .
$$

Equation (36) is plotted in Figure 6b along with the percent difference between it and equation (29). As the graph illustrates, equation (36) is within 5\% of equation (29) for $\Omega>3$. Under this condition, equation (36) will only be accurate when predicting larger values of $C V_{\dot{m}[2]}$ (e.g., $C V_{\dot{m}[2]}>0.7$ ). It would be advantageous to have a simplified screening-level equation for $C V_{\dot{m}[2]}=f(\Omega)$ applicable for $0.3 \leq C V_{\dot{m}[2]} \leq 0.7$. A lower 
limit of 0.3 was chosen to avoid complications associated with the local minima and maxima, and the non-unique nature of $C V_{\dot{m}[2]}=f(\Omega)$ as $\Omega$ is reduced. More importantly, predictions of uncertainty of this small magnitude are not considered appropriate for a screening-level analysis. An alternative, simplified equation that can be used to estimate $C V_{\dot{m}[2]}$ in the range $0.3<C V_{\dot{m}[2]}<0.7$ is

$C V_{\dot{m}[2]}=0.33 \Omega-0.25$ for $1.5 \leq \Omega \leq 3$,

obtained using linear regression in the region defined by $1.5 \leq \Omega \leq 3$, with $R^{2}=1.0$. Consequently, a simplified, screening-level equation for $C V_{\dot{m}[2]}$ is

$$
C V_{\dot{m}[2]}=\left\{\begin{array}{l}
<0.3 \text { for } \Omega<1.5 \\
0.33 \Omega-0.25 \text { for } 1.5 \leq \Omega \leq 3 \\
\sqrt{\frac{\Omega}{2}-1} \quad \text { for } \Omega>3
\end{array} .\right.
$$

At many sites, the vertical extent of the contaminant distribution is less than the transverse distribution (e.g., Guilbeault et al., 2005), reflecting differences in vertical and horizontal hydraulic conductivities. Moreover, Kübert and Finkel (2006) discussed the dependence of $\dot{m}$ measurement error on both the horizontal and vertical spacing relative to the horizontal and vertical $K$ correlation lengths, respectively, and noted that appropriate sample spacing was needed in both dimensions to see significant reductions in mean error. Figure 6 and the associated discussions above are based on $b / \sigma_{y}=d / \sigma_{z}$, but this condition may not be achieved with a given sampling network without a priori knowledge of $\sigma_{y}$ and $\sigma_{y}$. To explore the sensitivity of $C V_{\dot{m}[2]}$ to the condition when 
$b / \sigma_{y} \neq d / \sigma_{z}$, we introduce two more parameters that describe the cross-sectional aspect ratio of the plume: $\rho_{1}=\sigma_{z} / \sigma_{y}$, and the aspect ratio of the sampling network: $\rho_{2}=d / b$. It is noted that the condition of $b / \sigma_{y}=d / \sigma_{z}$ is equivalent to $\rho_{1}=\rho_{2}$, or $\rho_{2} / \rho_{1}=1$. Plotted in Figure 7 is $C V_{\dot{m}[2]}$ versus $\Omega$ for $\rho_{2} / \rho_{1}=\{0.1,0.2,0.5,1,2,5,10\}$, which was created using equation (29). Results for specific values of $\rho_{2} / \rho_{1}$ and its reciprocal plot on top of each other, which is to be expected given the symmetry of equation (29) with respect to $b / \sigma_{y}$ and $d / \sigma_{z}$. A minimum uncertainty occurs when $\rho_{2} / \rho_{1}=1$, which suggests an optimal condition when the aspect ratio of the sampling network equals the aspect ratio of the contaminant distribution. As the difference between $\rho_{1}$ and $\rho_{2}$ grows, the uncertainty increases, but this effect becomes less pronounced as $\Omega$ increases.

\section{Comparisons to Other Uncertainty Studies}

Case 1. Comparison to Li et al. (2007). Li et al. (2007) quantified uncertainty by estimating empirical $\dot{m}$ CDFs through joint simulation of random $C$ and $K$ fields, conditioned to field measurements. The approach was demonstrated using a hypothetical site based on subsurface characteristics of the Bachman Road site in Oscado, MI. Model simulations included DNAPL transport, and considered changing $\dot{m}$ from the DNAPL source zone due to partial DNAPL mass removal. Results were evaluated through an evaluation of the mean relative error and normalized $95 \%$ confidence interval. However, Figure 3 from Li et al. (2007) shows the $\dot{m}$ CDFs and lists a mean $\dot{m}=14.6 \mathrm{~g} / \mathrm{d}$ with a $C V=0.42$. These results were obtained using a sampling network with 24 sampling points uniformly distributed across a control plane with $\Lambda=77 \mathrm{~m}^{2}$, resulting in $S_{D}=$ 
$0.31 \mathrm{pts} / \mathrm{m}^{2}$. The true $\dot{m}$ was also given in the figure as $11.5 \mathrm{~g} / \mathrm{d}$. While the true $J_{\max }$ is unknown, data from their Figure 3 was used to estimate $J_{\max }=1.4 \mathrm{~g} / \mathrm{m}^{2} / \mathrm{d}$, assuming $I=$ 0.01 based on information provided in Christ et al. (2006). Therefore $\Omega=0.4$ using the true $\dot{m}$ and assuming $\rho_{2} / \rho_{1}=1$, which indicates $C V_{\dot{m}[2]}<0.3$ using equation (38), and from the screening-level analysis presented herein the sampling design is considered sufficient. Alternatively, $J_{\max }$ necessary to yield $C V=0.42$ can be calculated from Equation (38): $J_{\max }=7.2 \mathrm{~g} / \mathrm{m}^{2} / \mathrm{d}$, which is not an unreasonable estimate given the potential range in the maximum $C$ and $q$. For example, this $J_{\max }$ value could be obtained assuming a maximum $C=22 \mathrm{mg} / \mathrm{L}$ and a maximum $q$ based on the mean $q$ plus one standard deviation (i.e., $0.33 \mathrm{~m} / \mathrm{d}$ ).

This comparison illustrates the sensitivity of predicting uncertainty using estimates of $\dot{m}$ and $J_{\max }$. From the perspective of a preliminary screening-level analysis, it is more likely that order-of-magnitude estimates are available for $\dot{m}$ and $J_{\max }$ . Assuming information is available for order-of-magnitude estimates of $\dot{m}=\{10,100\}$ $\mathrm{g} / \mathrm{d}$ and $J_{\text {max }}=\{1,10\} \mathrm{g} / \mathrm{m}^{2} / \mathrm{d}$, then $\Omega=\{0.03,0.32,0.32,3.22\}$ using $S_{D}=0.31 \mathrm{pts} / \mathrm{m}^{2}$. In the first three cases, $C V_{i[2]}<0.3$ based on equation (38), and it would be concluded that the sampling design is sufficient from a screening-level analysis. In the last case (which corresponds to the event that $\dot{m}=10 \mathrm{~g} / \mathrm{d}$ and $J_{\max }=10 \mathrm{~g} / \mathrm{m}^{2} / \mathrm{d}$ ), $C V_{\dot{m}[2]}=0.8$ using equation (38). Therefore, only in this case would uncertainty be sufficiently large to potentially warrant concern from a screening-level analysis, depending on the given sitespecific application for which $\dot{m}$ is being measured. Selecting a sampling network with 
a larger sampling density would have to weigh the likelihood of this particular case against the cost of over design. Or, consideration could be given to a staged sampling approach (e.g., Li and Abriola, 2009), so that the risk this particular case represents is addressed through additional measurements if needed. We emphasize that defining $C V_{i m[2]}<0.3$ as acceptable and $C V_{i m[2]}=0.8$ as questionable levels of uncertainty was done here for illustration purposes only. Defining what constitutes an acceptable or unacceptable level of uncertainty should be based on the intended use of the $\dot{m}$ measurements, and these definitions may vary from one application to another.

Case 2. Comparison to Klammler et al. (2012). Klammler et al. (2012) present a stochastic simulation method to estimate the uncertainty of $\dot{m}$ estimates based on PFM measurements, and the method was applied to the pre-treatment $\dot{m}$ estimate from Nonaqueous Phase Liquid (NAPL) Area 1 of the Fort Lewis Logistics Center Superfund site, near Tacoma, Washington. The PFM measurements were presented in Brooks et al. (2008) as part of a study that evaluated changes in $\dot{m}$ due to source-zone treatment. The analysis completed by Klammler et al. (2012) yielded a mean $\dot{m}=777 \mathrm{~g} / \mathrm{d}$ and $C V=0.16$ using a sampling network with an average $S_{D}=0.4 \mathrm{pts} / \mathrm{m}^{2}$. Brooks et al. (2008) used a Thiessen polygon method with the same data set and estimated $\dot{m}=743 \mathrm{~g} / \mathrm{d}$ assuming groundwater flow perpendicular to the control plane, and $646 \mathrm{~g} / \mathrm{d}$ accounting for the nonorthogonal flow direction relative to the control plane. Using $\dot{m}=743 \mathrm{~g} / \mathrm{d}, J_{\max }=18$ $\mathrm{g} / \mathrm{m}^{2} / \mathrm{d}$ (Figure 5, Brooks et al., 2008), and assuming $\rho_{1} / \rho_{2}=1, C V_{\dot{m}[2]}<0.3$ using Equation (38). This estimate is insensitive to changes in $\dot{m}$ from 646 to $777 \mathrm{~g} / \mathrm{d}$.

A comparison of these results (i.e., 0.16 versus $<0.30$ ) highlights again the perspective that should be used with screening-level estimates of uncertainty, which is 
that screening-level estimates help guide sampling design decisions prior to field measurements, but they should not replace more rigorous post-measurement analyses. The application above used the measured values of $\dot{m}$ and $J_{\text {max }}$ to estimate $C V$, which of course were not available prior to collecting the measurements. If sufficient information had been available to estimate order of magnitude ranges of $\dot{m}=\{100,1000\} \mathrm{g} / \mathrm{d}$ and $J_{\text {max }}=\{10,100\} \mathrm{g} / \mathrm{m}^{2} / \mathrm{d}$, then application of equation (38) would have yielded $C V=$ $\{<0.30,<0.30,<0.30,0.58\}$. Consequently, this sampling design may be evaluated as sufficient for all but the case of $\dot{m}=100 \mathrm{~g} / \mathrm{d}$ and $J_{\max }=100 \mathrm{~g} / \mathrm{m}^{2} / \mathrm{d}$, depending on the given application. Selecting a sampling network with a larger sampling density would again have to weigh the likelihood of this particular case against the cost of over design.

Case 3. Comparison to Mackay et al. (2012). The field-based evaluation of $\dot{m}$ uncertainty reported by Mackay et al. (2012) was completed using a controlled release experiment of a bromide tracer at the Vandenberg Air Force Base, Santa Barbara, California. Mackay et al. (2012) reported uncertainty using relative percent difference $(R P D)$, where measurements using sparse sampling densities were compared to the measurement completed with the most dense network, which had $S_{D}=1.4 \mathrm{pts} / \mathrm{m}^{2}$. The sparse networks resulted in $S_{D}=\{0.20,0.24,0.28,0.35,0.47,0.70\} \mathrm{pts} / \mathrm{m}^{2}$. Bromide was injected at a rate of $122 \mathrm{~g} / \mathrm{d}$, using a total flowrate of $0.576 \mathrm{~m}^{3} /$ day, therefore the average injected bromide $C$ was $\sim 212 \mathrm{~g} / \mathrm{m}^{3}$ (mg/L). The aquifer was reported to be moderately heterogeneous, and the site-wide estimate of groundwater velocity was reported to be in the range of 0.53 to $0.75 \mathrm{~m} / \mathrm{d}$. Assuming an effective porosity of 0.34 , an estimate of the maximum $q$ is $0.26 \mathrm{~m} /$ day. Consequently the estimate of the maximum 
bromide flux is $55 \mathrm{~g} / \mathrm{m}^{2} /$ day. For $S_{D}=\{0.20,0.24,0.28\} \mathrm{pts} / \mathrm{m}^{2}, \Omega=\{2.24,1.87$, $1.60\}$, and application of equation (33) yields $C V_{\dot{m}}=\{0.77,0.57,0.36\}$. The maximum $R P D$ values reported by Mackay et al. (2012) for these cases were $\sim 1.45, \sim 1.05$, and 20.90. Of course, there is no expectation for $C V_{\dot{m}}$ to equal the maximum $R P D$, but information was not available to calculate the actual measured $C V_{\dot{m}}$ from the study. Consequently, a qualitative comparison is made between $C V_{\dot{m}}$ and $R P D$. Another important distinction to note when comparing the analytical results developed herein to the field-based results of Mackay et al. (2012) is that bromide was injected into two wells creating a bimodal contaminant pattern, versus the single peak flux assumed herein.

\section{SUMMARY AND CONCLUSIONS}

The uncertainty of mass discharge measurements associated with point-scale measurement methods under simplified conditions was investigated by deriving analytical solutions for the mass discharge coefficient of variation. Two conceptual models were used. In the first case, a depth-averaged system was assumed, resulting in a two-dimensional system consisting of one-dimensional groundwater flow perpendicular to a one-dimensional control plane of uniformly-spaced sampling points. The contaminant flux along the control plane was assumed to be normally distributed. In the second case, a three-dimensional system was assumed consisting of one-dimensional groundwater flow perpendicular to a two-dimensional control plane of uniformly spaced sampling points. The contaminant flux in this case was assumed to be distributed according to the bivariate normal distribution. The center point for the flux distributions in both cases was allowed to vary in the domain of the control plane as a uniform random 
variable. Simplified equations for the uncertainty were investigated to facilitate screening-level evaluations of uncertainty as a function of sampling network design. Results were used to express uncertainty as a function of the length of the control plane and number of wells, or alternatively as a function of the sample spacing.

Uncertainty was also expressed as a function of a new dimensionless parameter, $\Omega$, defined as the ratio of the maximum local flux to the product of mass discharge and sample density. Expressing uncertainty as a function of $\Omega$ provided a convenient means to demonstrate the dependence of uncertainty on the magnitude of a local hot spot, the magnitude of mass discharge across the control plane, the distribution of the contaminant across the control plane, and the sampling density. Results agree with other published works about the dependence of uncertainty on sampling density. They also agree with published works about the dependence of uncertainty on the magnitude of mass discharge, but that dependence must also consider the magnitude of the flux. Comparing two cases with different mass discharge, but equal flux, the case with the smaller mass discharge would require a larger sampling density to ensure an equal level of certainty between the cases. However, if both the mass discharge and flux changed proportionally, then an equal level of uncertainty is obtained for both cases using a single sampling density. Therefore, while general recommendations for sampling density are useful for screening-level guidelines, they should be used with the recognition that the required sampling density to achieve a given level of certainty depends not just on the level of mass discharge, but on the inter-relationship between mass discharge, the local maximum flux, and the resulting distribution of flux across the control plane. 
Predicting uncertainty with the screening-level equations presented herein requires some knowledge of site conditions, or more specifically, some knowledge of the very characteristic that is to be measured. Developing an accurate conceptual model of a site is an iterative process built upon measurements of various subsurface properties collected over time, and the certainty of the conceptual model should improve as more data is collected. Likewise, the measurement of mass discharge could be viewed from the same perspective. Preliminary estimates of mass discharge are made given current knowledge of subsurface characteristics, and the uncertainty of those estimates is likewise evaluated based on the current information. If the level of uncertainty is unacceptable, then the current knowledge is used to design field tests to collect more data, and the process is then repeated.

\section{ACKNOWLEDGEMENTS}

The work upon which this paper is based was supported by the U.S. Environmental Protection Agency through its Office of Research and Development with funding provided by the Strategic Environmental Research and Development Program (SERDP), a collaborative effort involving the U.S. Environmental Protection Agency (EPA), the U.S. Department of Energy (DOE), and the U.S. Department of Defense (DoD). It has not been subjected to Agency review and, therefore, does not necessarily reflect the views of the Agency and no official endorsement should be inferred. The authors thank Pat Bush, Senior Environmental Employee, for her editorial review; and Kathy Tynsky, SRA International, Inc., for her assistance with the figures. Comments 
from two anonymous reviewers were used to improve the manuscript, and we are grateful to them for their reviews.

\section{REFERENCES}

Annable, M.D., Hatfield, K., Cho, J., Klammler, H., Parker, B.L., Cherry, J.A., Rao, P.S.C., 2005. Field-Scale Evaluation of the Passive Flux Meter for Simultaneous Measurement of Groundwater and Contaminant Fluxes. Environmental Science \& Technology 39 (18), 7194-7201.

Basu, N.B., Rao, P.S.C., Poyer, I.C., Annable, M.D., Hatfield, K., 2006. Flux-based assessment at a manufacturing site contaminated with trichloroethylene. Journal of Contaminant Hydrology 86 (1-2), 105-127.

Basu, N.B., Rao, P.S.C., Falta, R.W., Annable, M.D., Jawitz, J.W., Hatfield, K., 2008. Temporal evolution of DNAPL source and contaminant flux distribution: Impacts of source mass depletion. Journal of Contaminant Hydrology 95 (3-4), 93-109.

Basu, N.B., Rao, P.S.C., Poyer, I.C., Nandy, S., Mallavarapu, M., Naidu, R., Davis, G.B., Patterson, B.M., Annable, M.D., Hatfield, K., 2009. Integration of traditional and innovative characterization techniques for flux-based assessment of dense nonaqueous phase liquid (DNAPL) sites. Journal of Contaminant Hydrology 105 (34), 161-172.

Bayer-Raich, M., Jarsjö, J., Liedl, R., Ptak, T., Teutsch, G., 2004. Average contaminant concentration and mass flow in aquifers from time-dependent pumping well data: analytical framework. Water Resources Research 40, W08303, doi:10.1029/2004WR003095. 
Bayer-Raich, M., Jarsjö, J., Liedl, R., Ptak, T., Teutsch, G., 2006. Integral pumping test analysis of linearly sorbed groundwater contaminants using multiple wells: Inferring mass flows and natural attenuation rates. Water Resources Research 42, W08411, doi:10.1029/2005WR004244.

Béland-Pelletier, C., Fraser, M., Barker, J., Ptak, T., 2011. Estimating contaminant mass discharge: A field comparison of the multilevel point measurement and the integral pumping investigation approaches and their uncertainties. Journal of Contaminant Hydrology 122 (1-4), 63-75.

Bockelmann, A., Ptak, T., Teutsch, G., 2001. An analytical quantification of mass fluxes and natural attenuation rate constants at a former gasworks site. Journal of Contaminant Hydrology 53 (3-4), 429-453.

Borden, R.C., Daniel, R.A., LeBrun IV, L.E., Davis, C.W., 1997. Intrinsic biodegradation of MTBE and BTEX in a gasoline-contaminated aquifer. Water Resources Research 33 (5), 1105-1115.

Brooks, M.C., Wood, A.L., Annable, M.D., Hatfield, K., Cho, J., Holbert, C., Rao, P.S.C., Enfield, C.G., Lynch, K., Smith, R.E., 2008. Changes in Contaminant Mass Discharge from DNAPL Source Mass Depletion: Evaluation at Two Field Sites. Journal of Contaminant Hydrology 102 (1-2), 140-153.

Brusseau, M.L., Nelson, N.T., Zhang, Z., Blue, J.E., Rohrer, J., Allen, T., 2007. Sourcezone characterization of a chlorinated-solvent contaminated Superfund site in Tucson, AZ. Journal of Contaminant Hydrology 90 (1-2), 21-40. 
Cai, Z., Wilson, R.D., Cardiff, M.A., Kitanidis, P.K., 2011. Increasing confidence in mass discharge estimates using geostatistical methods. Ground Water 49 (2), 197 208.

Cai, Z., Wilson, R.D., Lerner, D.N., 2012. Assessing TCE source bioremediation by geostatistical analysis of a flux fence. Ground Water 50 (6), 908-917.

Chapman, S.W., Parker, B.L., 2005. Plume persistence due to aquitard back diffusion following dense nonaqueous phase liquid source removal or isolation. Water Resources Research 41, W12411, doi:10.1029/2005WR004224.

Chen, X., Brooks, M.C., Wood, A.L., 2014. The uncertainty of mass discharge measurements using pumping methods under simplified conditions. Journal of Contaminant Hydrology 156, 16-26.

Christ, J.A., Ramsburg, C.A., Pennell, K.D., Abriola, L.M., 2006. Estimating mass discharge from DNAPL source zones using upscaled mass transfer coefficients: An evaluation using multiphase numerical simulations, Water Resources Research 42, W11420, doi:10.1029/2006WR004886.

DiFilippo, E.L., Brusseau, M.L., 2008. Relationship between mass-flux reduction and source-mass removal: Analysis of field data. Journal of Contaminant Hydrology $98(1-2), 22-35$.

Einarson, M.D., Mackay, D.M., 2001. Predicting impacts of groundwater contamination. Environmental Science \& Technology 35 (3), 66A-73A.

Fraser, M., Barker, J.F., Butler, B., Blaine, F., Joseph, S., Cooke, C., 2008. Natural attenuation of a plume from an emplaced coal tar creosote source over 14 years. Journal of Contaminant Hydrology 100 (3-4), 101-115. 
Freitas, J.G., Mocanu, M.T., Zoby, J.L.G., Molson, J.W., Barker, J.F., 2011. Migration and fate of ethanol gasoline in groundwater: A modeling analysis of a field experiment. Journal of Contaminant Hydrology 119 (1-4), 25-43.

Goltz, M.N., Close, M.E., Yoon, H., Huang, J., Flintoft, M.J., Kim, S., Enfield, C., 2009. Validation of two innovative methods to measure contaminant mass flux in groundwater. Journal of Contaminant Hydrology 106 (1-2), 51-61.

Guilbeault, M.A., Parker, B.L., Cherry, J.A., 2005. Mass and flux distributions from DNAPL zones in sandy aquifers. Ground Water 43 (1), 70-86.

Hatfield, K., Annable, M.D., Cho, J., Rao, P.S.C., Klammler, H., 2004. A direct passive method for measuring water and contaminant fluxes in porous media. Journal of Contaminant Hydrology 75 (3-4), 155-181.

Holder, TH., Teutsch, G., Ptak, T., Schwarz, R., 1998. A new approach for source zone characterization: the Neckar Valley study. Groundwater Quality: Remediation and Protection, Proceedings of the GQ'98 Conference, Tübingen, Germany, IAHS Publication number 250, 49-55.

ITRC (Interstate Technology \& Regulatory Council), 2010. Use and Measurement of Mass Flux and Mass Discharge. MASSFLUX-1. Washington, D.C.: Interstate Technology \& Regulatory Council, Integrated DNAPL Site Strategy Team. www.itrcweb.org.

Kao, C.M., Prosser, J., 2001. Evaluation of natural attenuation rate at a gasoline spill site. Journal of Hazardous Materials 82 (3), 275-289.

Kao, C.M., Wang, C.C., 2000. Control of BTEX migration by intrinsic bioremediation at a gasoline spill site. Water Research 34 (13), 3413-3423. 
Kao, C.M., Wang, Y.S., 2001. Field investigation of the natural attenuation and intrinsic biodegradation rates at an underground storage tank site. Environmental Geology $40(4-5), 622-630$.

Kavanaugh, M., Deeb, R., Hawley, E., 2011. Diagnostic Tools for performance evaluation of innovative in-situ remediation technologies at chlorinated solventcontaminated sites, Final Report for ESTCP Project ER-200318; Environmental Security Technology Certification Program. http://www.serdp-estcp.org/ProgramAreas/Environmental-Restoration/Contaminated-Groundwater/PersistentContamination/ER-200318.

King, M.W.G., Barker, J.F., Devlin, J.F., Butler, B.J., 1999. Migration and natural fate of a coal tar creosote plume, 2. Mass balance and biodegradation indicators. Journal of Contaminant Hydrology 39 (3-4), 281-307.

Klammler, H., Hatfield, K., da Luz, J.A.G., Annable, M.D., Newman, M., Cho, J., Peacock, A., Stucker, V., Ranville, J., Cabaniss, S.A., and Rao, P.S.C., 2012. Contaminant discharge and uncertainty estimates from passive flux meter measurements. Water Resources Research 48, W02512, doi:10.1029/2011WR010535.

Kübert, M., Finkel, M., 2006. Contaminant Mass Discharge Estimation in Groundwater Based on Multi-level Point Measurements: A Numerical Evaluation of Expected Errors. Journal of Contaminant Hydrology 84 (1-2), 55-80.

Li, K.B., Abriola, L.M., 2009. A multistage multicriteria spatial sampling strategy for estimating contaminant mass discharge and its uncertainty. Water Resources Research 45, W06407, doi:10.1029/2008WR007362. 
Li, K.B., Goovaerts, P., Abriola, L.M., 2007. A geostatistical approach for quantification of contaminant mass discharge uncertainty using multilevel sampler measurements, Water Resources Research 43, W06436, doi:10.1029/2006WR005427.

MacKay, D.M., Einarson, M.D., Kaiser, P.M., Nozawa-Inoue, M., Goyal, S., Chakraborty, I., Rasa, E., Scow, K.M., 2012. Mass discharge in a tracer plume: evaluation of the Theissen polygon method. Ground Water, doi: 10.1111/j.17456584.2012.00912.x.

Newell, C.J., Farhat, S.K., Adamson, D.T., Looney, B.B., 2011. Contaminant plume classification system based on mass discharge. Ground Water 49 (6), 914-919.

Rein, A., Bauer, S., Dietrich, P., Beyer, C., 2009. Influence of temporally variable groundwater flow conditions on point measurements and contaminant mass flux estimations. Journal of Contaminant Hydrology 108 (3-4), 118-133.

Schwartz, R., Ptak, T., Holder, T., Teutsch, G., 1998. Groundwater risk assessment at contaminated sites: a new investigation approach. Groundwater Quality: Remediation and Protection, Proceedings of the GQ'98 Conference, Tübingen, Germany, IAHS Publication number 250, 49-55.

Schwede, R. L., Cirpka, O.A., 2010. Stochastic evaluation of mass discharge from pointlike concentration measurements, Journal of Contaminant Hydrology 111 (14), 36-47.

Semprini, L., Kitanidis, P.K., Kampbell, D.H., Wilson, J.T., 1995. Anaerobic transformation of chlorinated aliphatic hydrocarbons in a sand aquifer based on spatial chemical distributions, Water Resources Research 31 (4), 1051-1062. 
Suarez, M.P., Rifai, H.S., 2002. Evaluation of BTEX remediation by natural attenuation at a coastal facility. Ground Water Monitoring and Remediation 22 (1), 62-77.

Suthersan, S., Divine, C., Quinnan, J., Nichols, E., 2010. Flux-informed remediation decision making, Ground Water Monitoring and Remediation 30 (1), 34-43.

Troldborg, M., Nowak, W., Tuxen, N., Bjerg, P.L., Helmig, R., Binning, P.J., 2010. Uncertainty evaluation of mass discharge estimates from a contaminated site using a fully bayesian framework. Water Resources Research 46, W12552, doi:10.1029/2010WR009227.

Troldborg, M., Nowak, W., Lange, I.V., Santos, M.C., Binning, P.J., Bjerg, P.L., 2012. Application of Bayesian geostatistics for evaluation of mass discharge uncertainty at contaminated sites. Water Resources Research 48, W09535, doi:10.1029/2011WR011785.

Wood, A.L., Annable, M.D., Jawitz, J.W., Falta, R.W., Brooks, M.C., Enfield, C.G., Rao, P.S.C., Goltz, M.N., 2009. Impacts of DNAPL source treatment: Experimental and modeling assessment of the benefits of partial DNAPL source removal, EPA 600/R-09/096, 149 pgs.

Page 40 of 48 


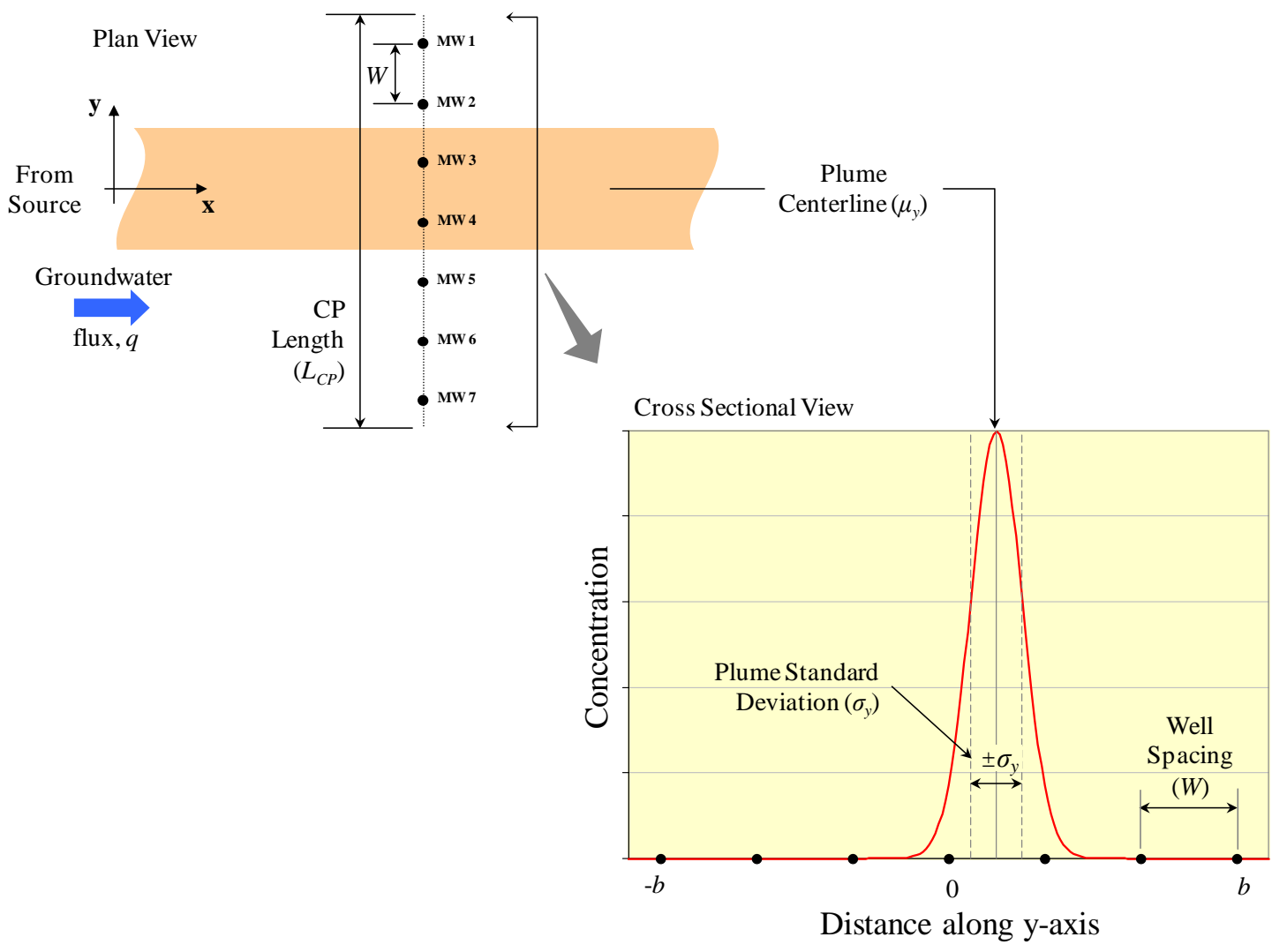

Figure 1. Plan and cross sectional views illustrating the conceptual depiction of a control plane with a one-dimensional contaminant distribution oriented perpendicular to groundwater flow (as adapted from Chen et al., 2014). 


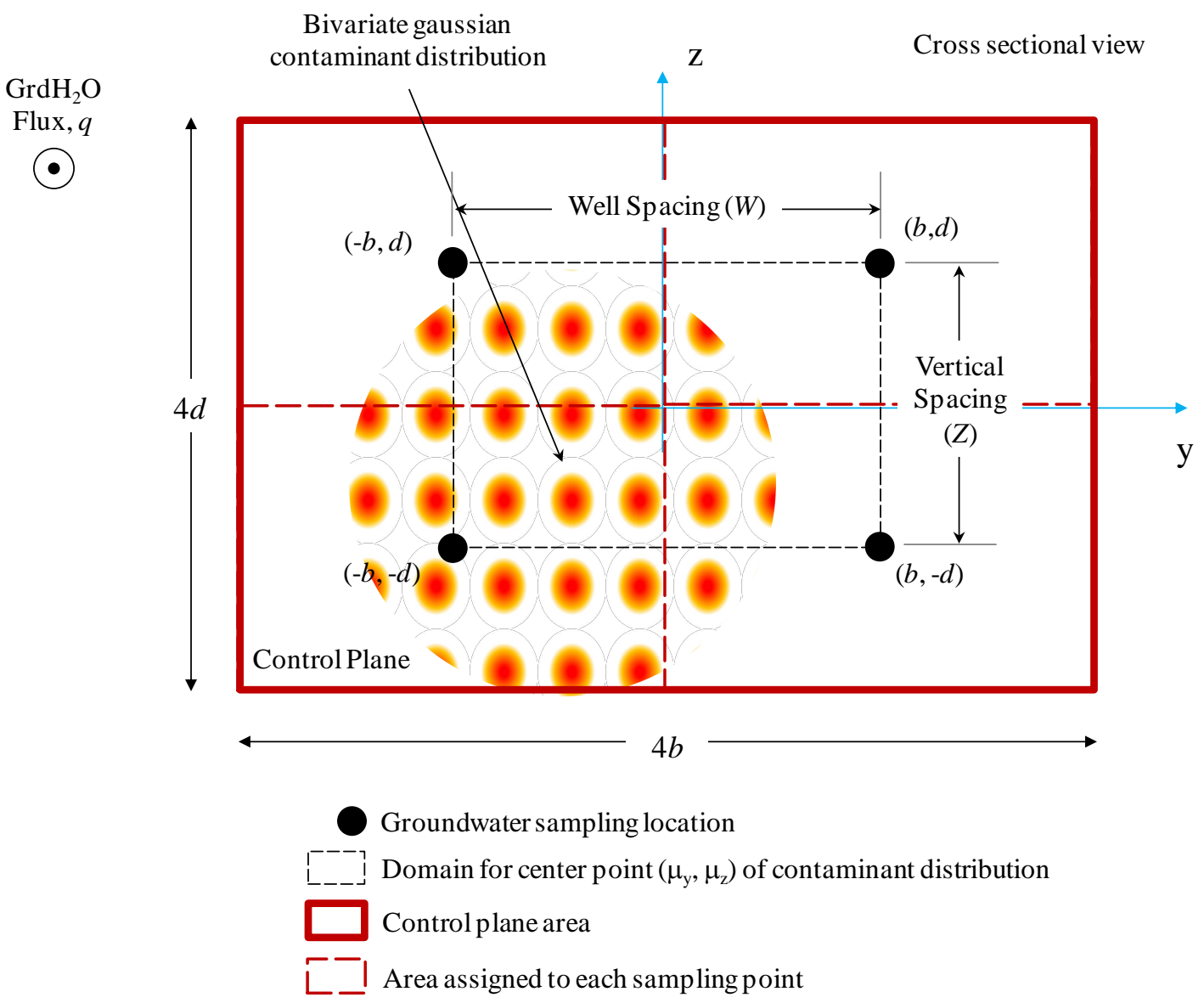

Figure 2. Cross-sectional view illustrating the conceptual depiction of a control plane with a two-dimensional contaminant distribution oriented perpendicular to groundwater flow.

Page 42 of 48 
a)

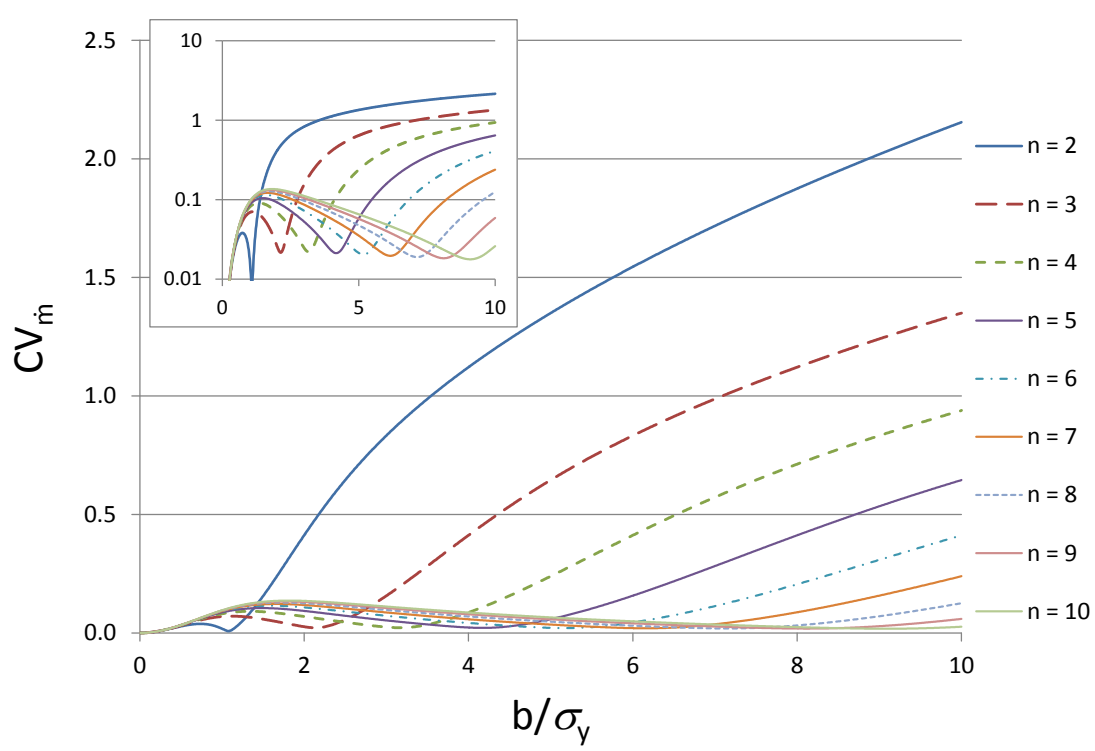

b)

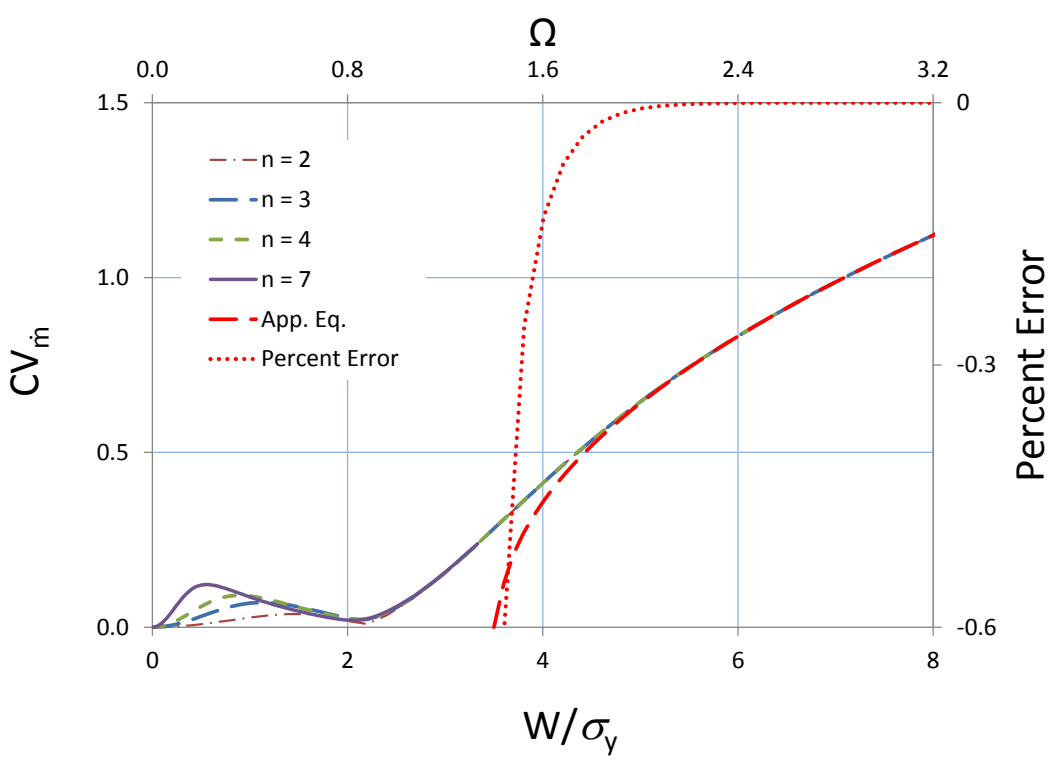

Figure 3. Results from the one-dimensional control plane analysis: a) mass discharge coefficient of variation $C V_{\dot{m}}$ versus $b / \sigma_{y}$ for $2 \leq n \leq 10$; b) $C V_{\dot{m}}$ versus $W / \sigma_{y}$ for $n=$ $\{2,3,4,7\}$. 

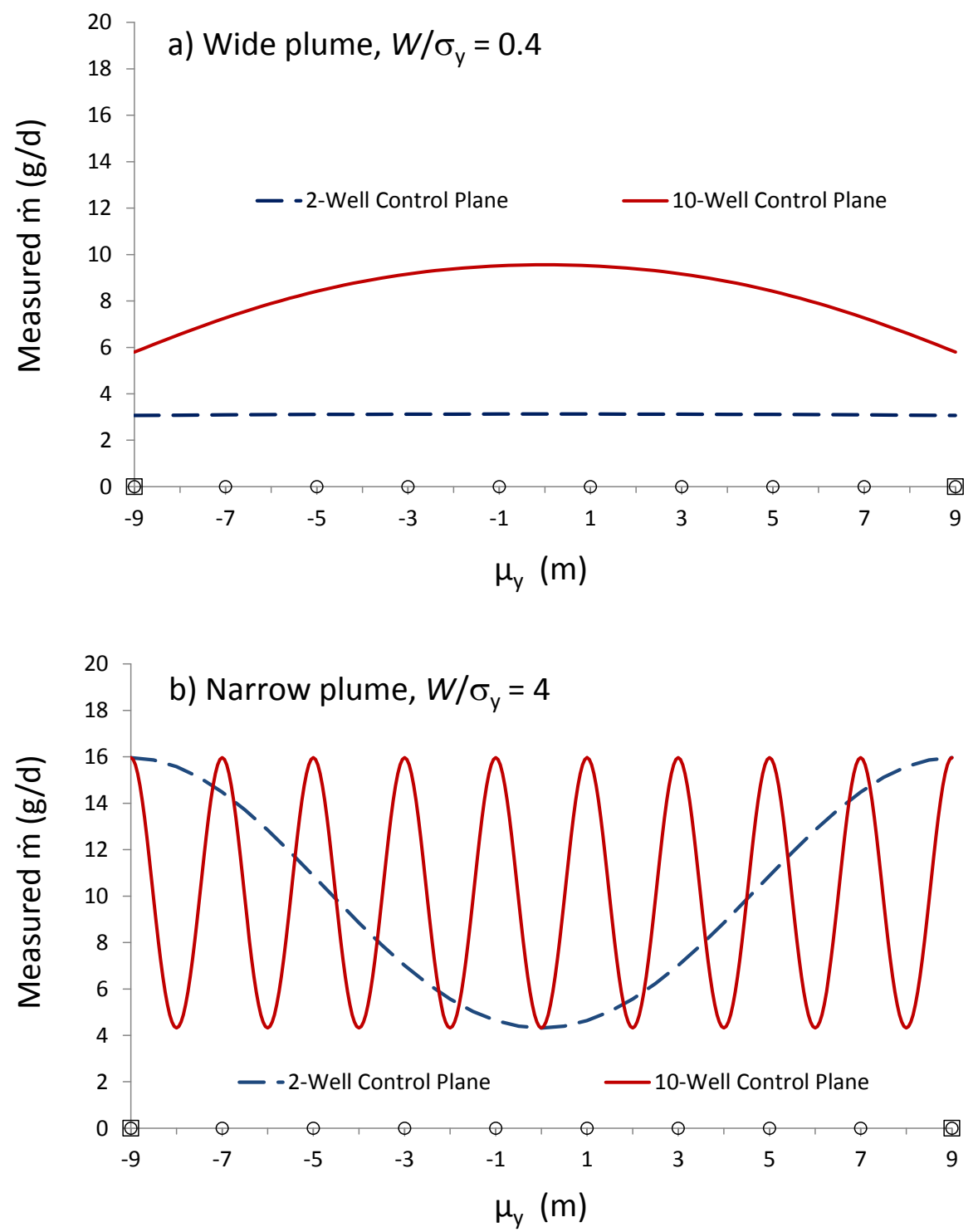

Figure 4. A comparison of measured $\dot{m}$ versus $\mu_{y}$ using control planes with 2 monitoring wells versus 10 monitoring wells for the one-dimensional control plane analysis. The open symbols along the $\mathrm{x}$-axis denote monitoring well locations: squares for the 2-well case and circles for the 10 -well case. In both cases $b=9 \mathrm{~m}$, and the wide plume is defined as $W / \sigma_{y}=0.4$ in panel (a) while the narrow plume is defined as $W / \sigma_{y}=$ 4 in panel (b). 
a)

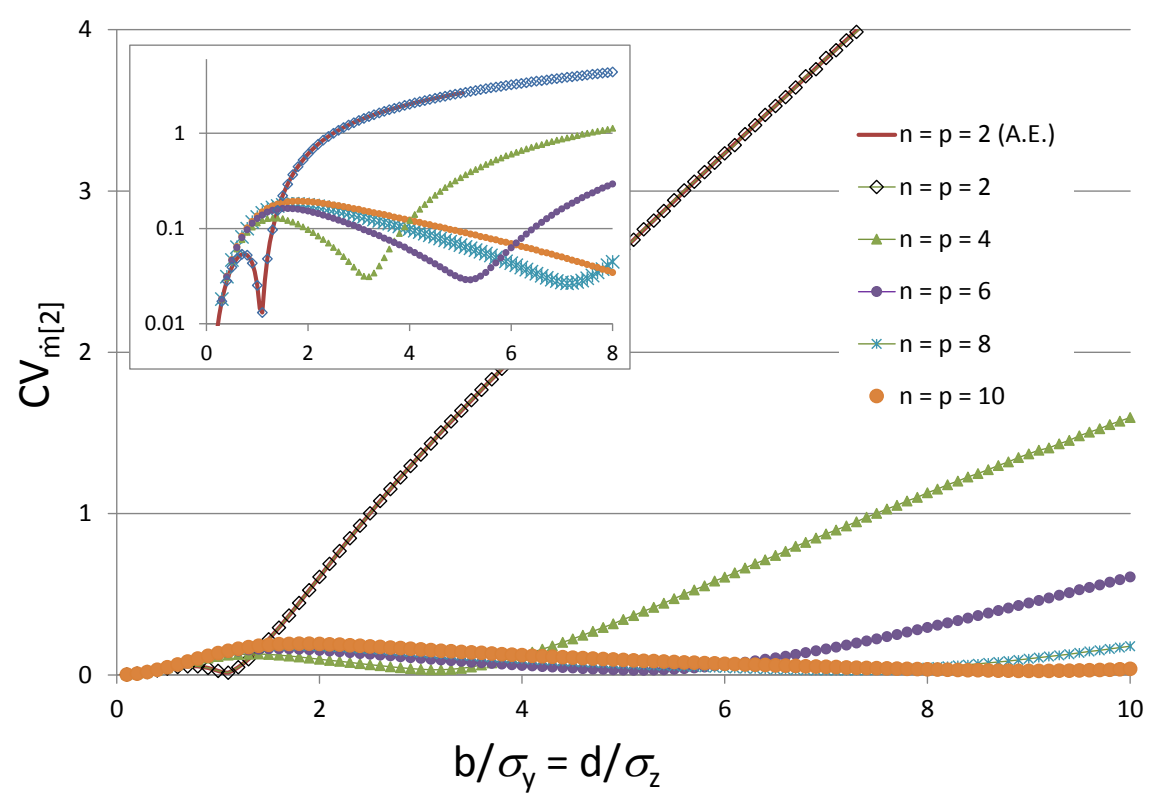

b)

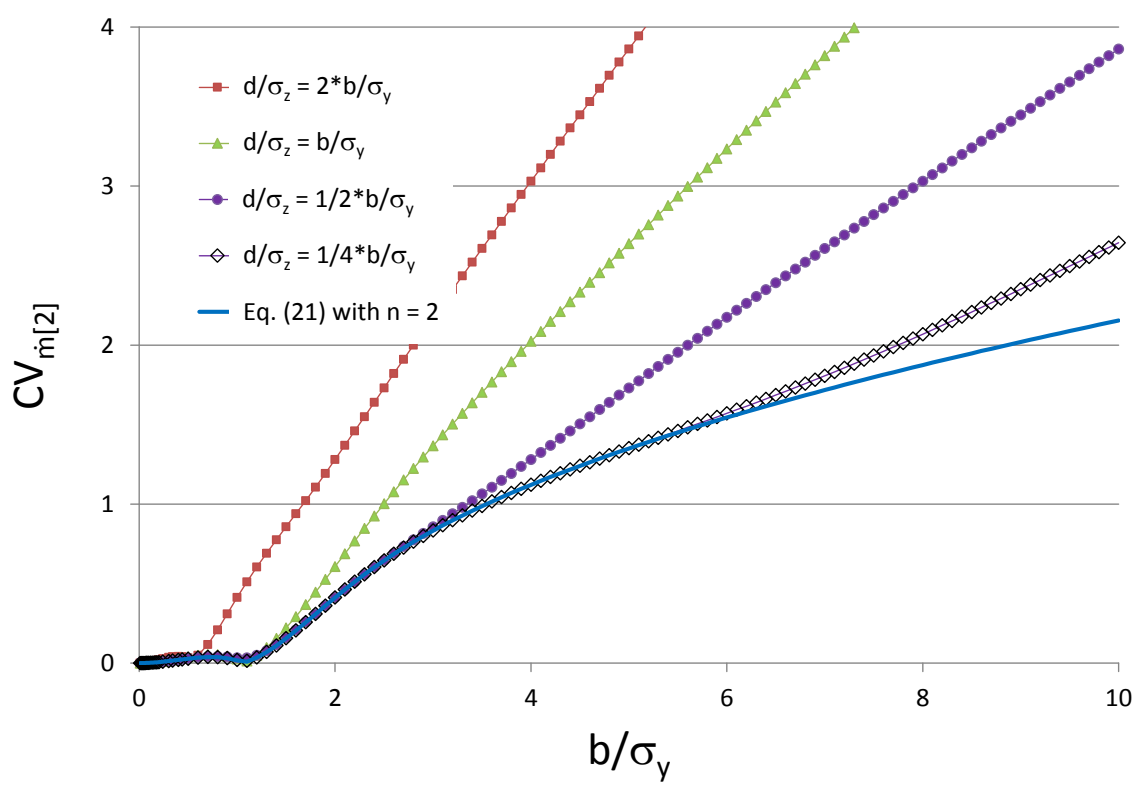

Figure 5. Results from the two-dimensional control plane analysis: a) mass discharge coefficient of variation $C V_{\dot{m}[2]}$ versus $b / \sigma_{y}=d / \sigma_{z}$, for $n=p=\{2,4,6,8,10\}$; b) $C V_{\dot{m}[2]}$ versus $b / \sigma_{y}$, for $d / \sigma_{z}=\left\{2 b / \sigma_{y}, b / \sigma_{y}, b / 2 \sigma_{y}, b / 4 \sigma_{y}\right\}$ and $n=p=2$, and $C V_{\dot{m}}$ for $n$ $=2$. 
a)

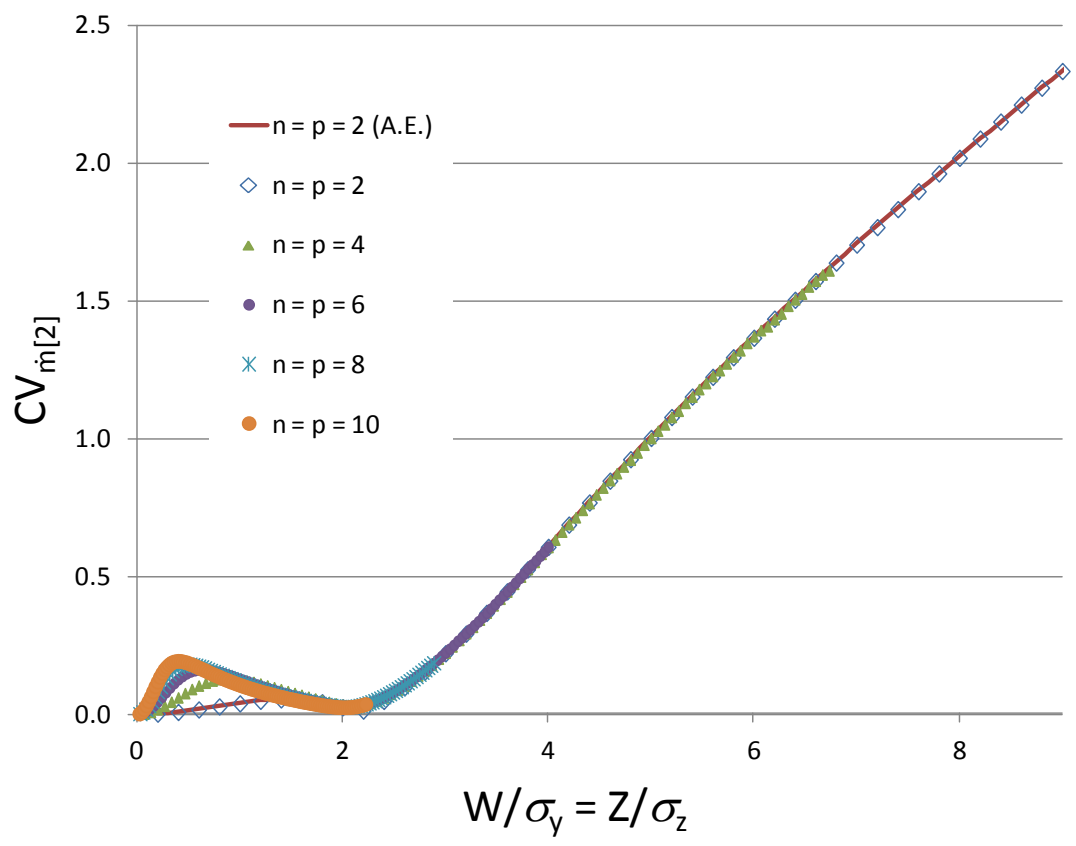

b)

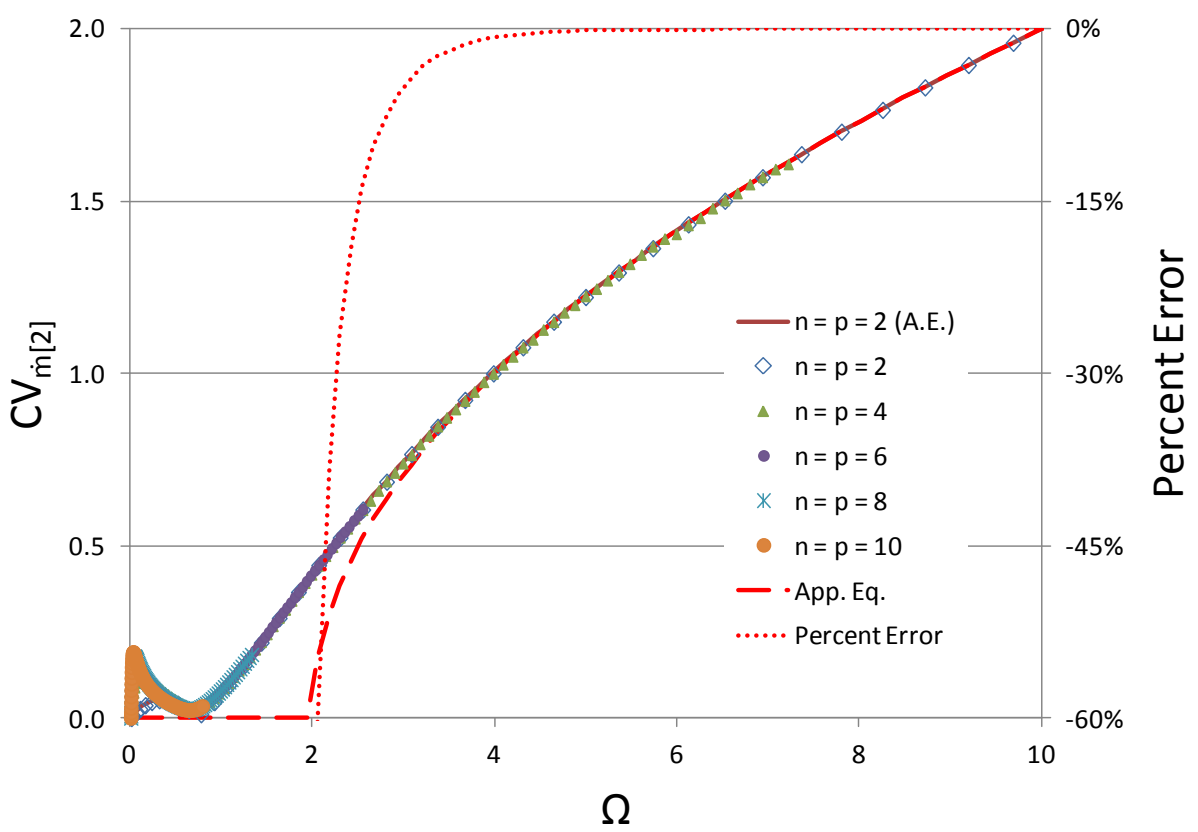

Figure 6. Results from the two-dimensional control plane analysis: a) mass discharge coefficient of variation $C V_{\dot{m}[2]}$ versus $W / \sigma_{y}=Z / \sigma_{z}$ for $n=p=\{2,4,6,8,10\}$; b) $C V_{\dot{m}[2]}$ versus the dimensionless factor $\Omega$. 




Figure 7. Mass discharge coefficient of variation for the two-dimensional control plane $C V_{\dot{m}[2]}$ versus the dimensionless factor $\Omega$, for $\rho_{2} / \rho_{1}=\{0.1,0.2,0.5,1,2,5,10\}$. 
Table 1. Comparison of results from the three cases presented in Section 4.

\begin{tabular}{l|c|c|c|c|c} 
Case & Reference & $\begin{array}{c}\text { Reported } \\
C V\end{array}$ & $\begin{array}{c}\text { Maximum } \\
\text { Reported } \\
R P D\end{array}$ & $\begin{array}{c}C V \text { from } \\
\text { Equation } \\
(38)\end{array}$ & $\begin{array}{c}C V \text { from } \\
\text { Equation } \\
(33)\end{array}$ \\
\hline 1 & Li et al. (2007) & 0.42 & & $<0.3$ & \\
\hline 2 & Klammler et al. (2012) & 0.16 & & $<0.3$ & \\
\hline 3 & Mackay et al. (2012) & & $\sim 1.45$ & & 0.77 \\
& & $\sim 1.05$ & & 0.57 \\
& & $\sim 0.9$ & & 0.36 \\
$C V=$ Coefficient of variation & & & & \\
$R P D=$ Relative percent difference
\end{tabular}

